\title{
Millimeter- and Terahertz-wave stochastic sensors based on reversible insulator-to-metal transition in vanadium dioxide
}

Fatemeh Qaderi ( $\sim$ fatemeh.qaderi@epfl.ch )

Ecole Polytechnique Federale de Lausanne (EPFL) https://orcid.org/0000-0001-8490-7475

Teodor Rosca

Ecole Polytechnique Federale de Lausanne (EPFL)

Maurizio Burla

ETH Zurich https://orcid.org/0000-0003-1189-4639

Juerg Leuthold

ETH Zurich https://orcid.org/0000-0003-0111-8169

Denis Flandre

Université catholique de Louvain

Adrian lonescu

EPFL https://orcid.org/0000-0003-2314-8887

\section{Article}

Keywords: low-energy photons, electromagnetic power sensors

Posted Date: January 6th, 2021

DOI: https://doi.org/10.21203/rs.3.rs-126738/v1

License: (c) (1) This work is licensed under a Creative Commons Attribution 4.0 International License.

Read Full License 


\title{
Millimeter- and Terahertz-wave stochastic sensors based on reversible insulator-to-metal transition in vanadium dioxide
}

\author{
Fatemeh Qaderi ${ }^{1,}{ }^{*}$, Teodor Rosca $^{1}$, Maurizio Burla ${ }^{2}$, Juerg Leuthold ${ }^{2}$, Denis Flandre ${ }^{3}$, \\ and Adrian M. Ionescu ${ }^{1, *}$
}

\author{
${ }^{1}$ Nanoelectronic devices laboratory (Nanolab), Department of Electrical Engineering, École polytechnique fédérale \\ de Lausanne (EPFL), Lausanne, 1015, Switzerland \\ ${ }^{2}$ ICTEAM, Ecole Polytechnique de Louvain (UCLouvain), Louvain-la-Neuve, 1348, Belgium \\ ${ }^{3}$ Institute of Electromagnetic Fields (IEF), Eidgenössische Technische Hochschule Zürich (ETHZ), Zürich, 8092, \\ Switzerland \\ "fatemeh.qaderi@epfl.ch \\ *adrian.ionescu@epfl.ch
}

\begin{abstract}
Sensitivity to low-energy photons in phase change materials enables the development of efficient millimeter-wave (mm-wave) and terahertz $(\mathrm{THz})$ detectors. Here, we present the concept of uncooled mm-wave detection based on the sensitivity of IMT threshold voltage to the incident wave by exploiting the characteristics of reversible insulator-to-metal transition (IMT) in Vanadium dioxide $\left(\mathrm{VO}_{2}\right)$ thin film devices. The detection concept is demonstrated through actuation of biased $\mathrm{VO}_{2} 2$-terminal switches encapsulated in a pair of coupled antennas on a $\mathrm{Si} / \mathrm{SiO}_{2}$ substrate. We also study the behavior of $\mathrm{VO}_{2}$ switches interrupting coplanar waveguide (CPW)s. Ultimately, we propose an electromagnetic wave-sensitive voltage-controlled spike generator based on the $\mathrm{VO}_{2}$ switches in an astable circuit. The fabricated sensors show record figs. of merit, such as responsivities of around $66.3 \mathrm{kHz} / \mathrm{mW}$ with a low noise equivalent power (NEP) of $20 \mathrm{nW}$ at room temperature, for a footprint of $2.5 \times 10^{-5} \mathrm{~mm}^{2}$, which can be easily scaled. This solution gives 3 times better responsivity with only $1 / 10$ footprint of the state of the art. However, the footprint is capable of being scaled down to few hundreds of nanometers. The responsivity in static measurements is $76 \mathrm{kV} / \mathrm{W}$ in the same circumstances. Based on experimental statistical data measured on robust fabricated devices, we investigate and report stochastic behavior and noise limits of $\mathrm{VO}_{2}$-based spiking sensors that are expected to form a new class of energy efficient transducers. The results highlight the capability of $\mathrm{VO}_{2}$ phase transition to serve for building electromagnetic power sensors, that can be triggered by low energy photons.
\end{abstract}

\section{Introduction}

$\mathrm{VO}_{2}$ is a strongly correlated electron material which undergoes a first-order IMT at $340 \mathrm{~K}$ from a low-temperature monoclinic insulating state to a high-temperature rutile metallic state, accompanied by structural, electrical, and optical changes ${ }^{1,2}$. The potential of IMT along with its sensitivity to external stimuli makes it promising for a variety of applications in resistive memories ${ }^{3-5}$, optical switches ${ }^{6,7}$, sensors ${ }^{8-10}$, tunable photonic devices ${ }^{11,12}$, brain-inspired and neuromorphic architectures ${ }^{13-15}$. We target the sensor application in the frequency range of $\mathrm{mm}$-wave and $\mathrm{THz}$. THz detectors are widely growing in different applications in spectroscopy ${ }^{16}$, astronomy ${ }^{17}$, biomedicine $^{18}$, imaging ${ }^{19}$, surveillance security ${ }^{20}$, high-data-rate communication ${ }^{21}$, etc.

Aside from thermal stimuli, the phase transition can be triggered by other perturbations, such as optical pumping or changes in electric field, pressure, and doping. The mechanism of electric-field-driven IMT has been under debate for a long time. Formation of conducting filaments at bias voltages above a threshold is explained in some studies ${ }^{22}$, while the mechanism of filamentation has been differently interpreted, such as the non-uniform current distribution followed by local Joule heating ${ }^{22-24}$, or a breakdown mechanism similar to the one in thin oxides $^{25}$. On the other hand, it has been argued that the electric field can induce IMT through nucleation of conducting filaments similar to the mechanism in chalcogenide-based phase-change memory and switches ${ }^{26}$ or without the need for a thermal process in Mott insulators ${ }^{27-29}$. The physics of such transitions is still under debate but it is recognized that it involves nucleation processes related to conductive path formation that are stochastic in nature $^{30}$.

Resistive switching in $\mathrm{VO}_{2}$ based on electric-field-assisted carrier generation, which leads to critical doping 
levels making the Mott insulator unstable, allows moderate fields to excite a large number of carriers with negligible heating ${ }^{31}$. The idea of electric-field-driven carrier generation is also reinforced by localized triggering of IMT by means of carbon nanotube (CNT)s to enhance the electric field locally and decreasing the required voltage for IMT in $\mathrm{VO}_{2}$ thin films ${ }^{32}$. Also, the feasibility of ultra-fast $\mathrm{VO}_{2}$ switching into a metastable metallic state ${ }^{33}$ indicates a tunneling mechanism, in which long-lived metallic domains are generated by intense multi-terahertz excitation. The rich dynamics of $\mathrm{VO}_{2}$ optical response to ultra-fast pulses makes it also capable of being used in efficient all-optical-controlled photonic systems ${ }^{34,35}$.

$\mathrm{VO}_{2}$ transition through illumination of IR photons using different wavelengths of optical pomping has shown the feasibility of photo-induced phase transition even by photons with smaller energy than the optical gap of $\mathrm{VO}_{2}$ $(0.6 \mathrm{eV})^{36}$. Considering the thermal requirements for transition between $\mathrm{M}_{1}$ and $\mathrm{R}$ phases $\left(2.32 \mathrm{eV} / \mathrm{nm}^{3}\right)$ calculated by integrating heat capacity and latent heat ${ }^{2}$, the fundamental process of photo-induced phase transition turns out to show a different mechanism from a complete thermally induced transition, and ultimately a density-driven mechanism is suggested for this transition ${ }^{36}$. This is essentially practical in the case of low-energy photons such as $\mathrm{THz}$ and mm-wave, as they cannot actuate carriers through the band-gap in a semiconductor.

In this work we design and experimentally demonstrate for the first time an ultrasensitive stochastic power sensor, by investigating the interaction of $\mathrm{mm}$-wave and $\mathrm{VO}_{2}$ at a controlled ambient in terms of voltage bias and temperature. The responsivity is at least 3 times larger than the similar sensors, along with an limit of detection (LoD) comparable to the state of the art, and a footprint of one order of magnitude smaller than the reported ones. We expect the same mechanism also for $\mathrm{THz}$ photons.

We will then report on the stochastic behavior of switching based on experimental statistical data measured on robust fabricated $\mathrm{VO}_{2}$ devices, using a spike oscillator readout. We particularly analyze the stochasticity features that translate in Poisson noise challenges for future designs.

We conduct a set of experiments on coupled antennas, having one antenna as an emitter and the other one as a receiver focusing mm-wave on a sensitive $\mathrm{VO}_{2}$ part. This is more concerning the proof of the detection concept at $\mathrm{mm}$-wave frequencies from a distance, along with the use of an antenna as a continuous-wave (CW) source.

In order to study $\mathrm{VO}_{2}$ electrical properties as well as the interaction of the material with mm-wave in a broadband range, we conduct the same experiments on a CPW interrupted by a $\mathrm{VO}_{2}$ slit. The experiments are generally based on the high-frequency actuation of $\mathrm{VO}_{2}$, assisted by a collective effect of a bias voltage.

Ultimately, we propose a readout circuit by means of an astable multi-vibrator formed of a $\mathrm{VO}_{2}$ impedance in series with an NMOS transistor (fig. 1(c)), in which the change in the frequency of oscillations represents the power applied to the $\mathrm{VO}_{2}$ sensor.

\section{Principle of $\mathrm{mm}$-wave and $\mathrm{THz}$ detection using $\mathrm{VO}_{2}$ IMT DC characteristics}

In this work we propose an electromagnetic power sensor based on tuning a 2-terminal $\mathrm{VO}_{2}$ device on which the high frequency radiation is concentrated by: - an adapted antenna design (fig. 1(a)), and, - directly applying a controlled power of high frequency signal to the device under test in a dedicated setup (fig. 1(b)).

In both configurations a heater (or a temperature reservoir) is used to tune the IMT transition point.

We exploit the collective effect of several excitation sources. By investigation of two-port $\mathrm{S}$ parameters and the static I-V characteristics of the $\mathrm{VO}_{2}$ switches, we probe the transition behavior in presence of an excitation signal at different power levels. According to the measured static current-voltage characteristics of $\mathrm{VO}_{2}$ 2-terminal switches, the IMT threshold voltage drops at elevated temperatures ${ }^{37}$. This gives the inspiration of combining a DC voltage with an external stimulus to implement the core of a sensing device. At a controlled temperature, the voltage bias makes $\mathrm{VO}_{2}$ sensitive to an incoming wave and the antennas give enhancement to the absorption. A general schematic of the setup for antenna measurements is illustrated in fig. 1(a), with high-frequency signal and the bias voltage de-coupled.

For broadband measurements, we used the simple structure of a CPW with a slit of $\mathrm{VO}_{2}$ in the middle. This way we could also inject the power more directly, so after the proof of concept by the antennas, in order to study the $\mathrm{VO}_{2}$ response to the high-frequency power with a higher resolution, we conducted static measurements on CPW-based devices. The simplified equivalent schematic for CPW experiments at a very low data rate is shown in fig. 1(b).

A practical and efficient sensing configuration proposed in this work, as depicted in fig. 1(c), is to connect the 2-terminal $\mathrm{VO}_{2}$ device in series with a conventional MOSFET and load a capacitor in their middle point, in order to build an astable oscillator. The frequency of oscillations in the output of the circuit in fig. 1(c) can be modulated by the power injected through the RF ports. According to fig. 1(d), the astability conditions are fulfilled at specific bias points where the transistor's current intersects that of the $\mathrm{VO}_{2}$ impedance immediately after IMT threshold, 
as experimentally demonstrated in supplementary materials.

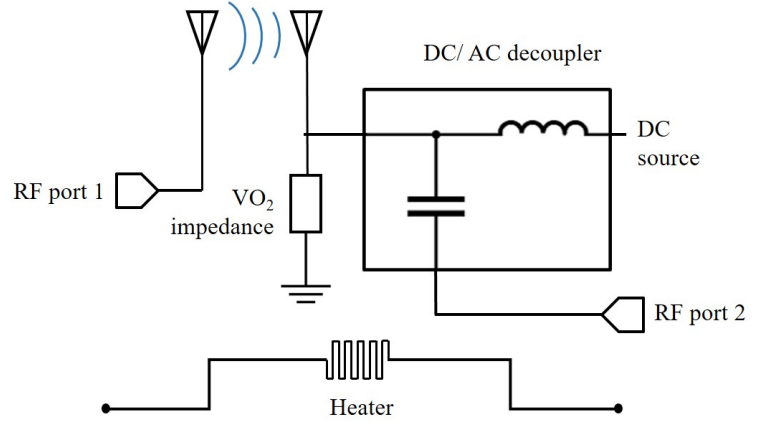

(a)

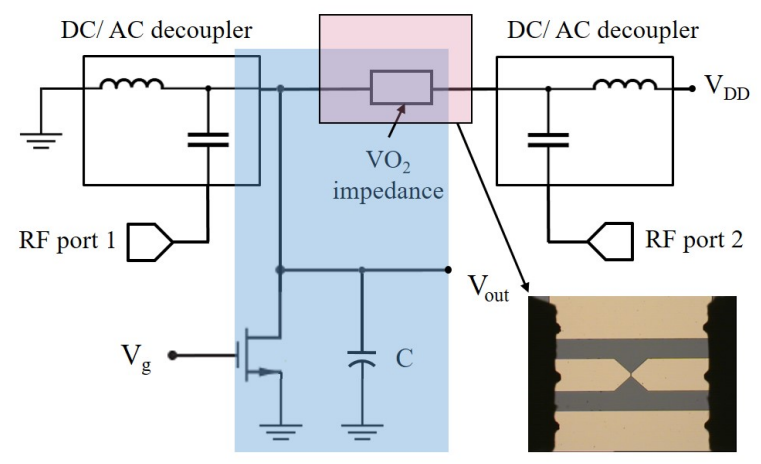

(c)

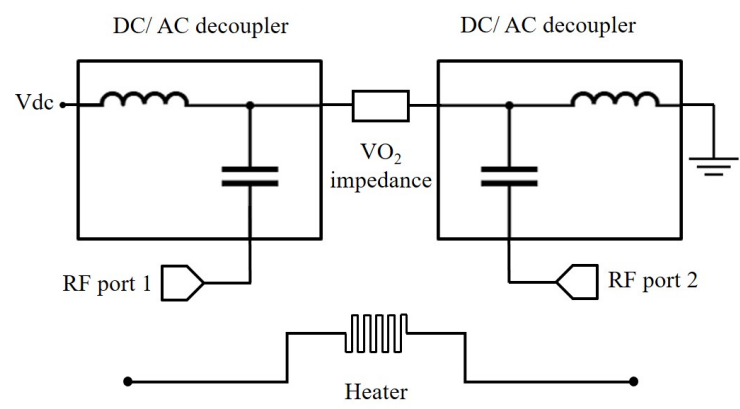

(b)

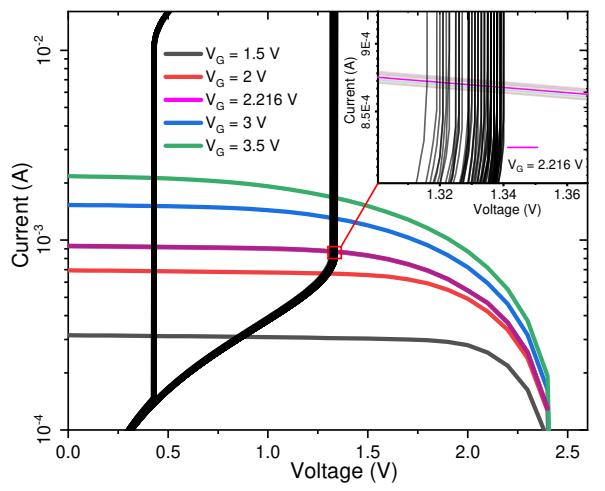

(d)

Figure 1. Depiction of the mm-wave detection and experimental setup used in this work. (a) $\mathrm{VO}_{2}$ in coupled antennas case, (b) $\mathrm{VO}_{2}$ in interrupted $\mathrm{CPW}$ case, (c) astable circuit using a 2-terminal $\mathrm{VO}_{2}$ device in interrupted $\mathrm{CPW}$ in series with a MOSFET, (d) the initial operation point of the oscillating sensor is set by the intersection of the static characteristics of $\mathrm{VO}_{2}$ and NMOS transistor at specific points supporting astability. Inset: the operational bias points and multiple cycles of experiments suggesting non-deterministic behavior.

\section{Results}

\section{$\mathrm{VO}_{2}$ actuation through coupled antennas}

By means of a pair of coupled antennas, we established an experimental scheme which can evaluate the $\mathrm{VO}_{2}$ actuation through electromagnetic radiation power. We have designed and fabricated a pair of dipole antennas through which the mm-wave power can be transmitted and received. At the receiver end, a 2-terminal $\mathrm{VO}_{2}$ device is encapsulated inside the antenna so that the incoming wave is concentrated there. A cross section of the middle part inside the receiving antenna as well as a microscopic image are illustrated in figs. 2(a) and (b), respectively. The zoomed parts of fig. 2(b) are taken by scaning electron microscopy (SEM), and the $\mathrm{VO}_{2}$ poly-crystalline grains are revealed in the last picture. The receiving antenna is biased after an AC/DC decoupler, at a voltage close to the one required for the transition. The closer we bias the antenna to the critical voltage, the more sensitive the IMT becomes to the radiation. The incident wave will add up to the threshold and actuate the metallic phase in $\mathrm{VO}_{2}$.

When $\mathrm{VO}_{2}$ is in insulating phase, the receiver antenna is terminated by the port from vector network analyzer (VNA) with a standard impedance, same as the emitter antenna. The $\mathrm{S}$ parameters over frequencies ranging from 40 to $220 \mathrm{GHz}$ are shown in figs. 2(c) and (d). The impedance matching at this phase is at its highest, and so does the transmission through the antennas. As soon as $\mathrm{VO}_{2}$ begins getting metallic, the termination becomes dominated by the smaller resistance of $\mathrm{VO}_{2}$ shunting the antenna. Therefore, the value of $\left|S_{21}\right|$ gets suppressed, as the mutual coupling vanishes by the change in the antenna configuration at the receiver. The receiver port 
matching and therefore the resonance in $\left|S_{11}\right|$ disappears as well, due to the dominant low impedance of $\mathrm{VO}_{2}$. A theoretical simulation of the $\mathrm{S}$ parameters by finite element method (FEM) for different values of $\mathrm{VO}_{2}$ conductivity is also in agreement with these results, provided in supplementary materials.

According to fig. $2(\mathrm{~d})$, the $\left|S_{21}\right|$ between the two ports is about $-11 \mathrm{~dB}$ at its highest. We consider it when estimating the absorbed power by the $\mathrm{VO}_{2}$-based antenna. Power loss also takes place through the cables, the transition from the infinity probes to the transmission line, the transition from the line to the dipole antenna, the return loss, and the reciprocal form of the mentioned transitions on the way to the receiving port. All the sources of errors from the tip of one RF probe until that of the other port affect the S parameters during the calibration, so we should compensate the cable and probe losses separately. So the upper bound of the received power can be estimated as:

$$
P_{R}=\left(P_{V N A}-P_{c}-P_{p}\right)\left|S_{21}\right|
$$

Where $P_{V N A}$ is the nominal power provided by the VNA, $P_{c}$ is the power loss by cables, and $P_{p}$ is the loss by infinity probes. According to the specifications in the frequency we use, the upper bound for the $\left|S_{21}\right|$ of the cables is collectively about $-4 \mathrm{~dB}$ and the upper bound for that of an infinity probe is about $-0.7 \mathrm{~dB}$. Having the $\left|S_{21}\right|$ between the antennas, the total transmission of power from VNA to the $\mathrm{VO}_{2}$ part of the antenna becomes $-15.7 \mathrm{~dB}$. At room temperature, even the maximum incident power to the $\mathrm{VO}_{2}$ antenna provided by the $\mathrm{VNA}$ is not sufficient to cause a transition, as illustrated in figs. 2(c) and (d); However, in presence of a bias voltage, the curves split dramatically by $\mathrm{VO}_{2}$ undergoing IMT due to radiation. The effect of IMT on $\left|S_{11}\right|$ and $\left|S_{21}\right|$ curve is shown in the same plots, respectively.

In the DC-characteristics of the $\mathrm{VO}_{2}$ sample of fig. 2(b), the IMT occurs near $1.713 \mathrm{~V}$. The power level of $0 \mathrm{dBm}$ at the VNA output (i.e. an effective power level of $-15.7 \mathrm{dBm}$ at the receiver), can split the curves for $\mathrm{VO}_{2}$ biased at any voltage higher than $1.690 \mathrm{~V}$. Closer to IMT critical voltage, for example at $1.695 \mathrm{~V}$, the minimum power required to make the transition reduces to $-10 \mathrm{dBm}$ at VNA output (effectively $-25.7 \mathrm{dBm}$ at the receiver). Right after experiencing IMT, without changing any circumstances, we set the mm-wave power back to a low level $(-30 \mathrm{dBm}$ at VNA output) which previously could not afford a transition, and observed that the curves remain at the metallic state values. This is in complete agreement with the hysteretic characteristics of the transition. It is worth noting that by removal of the bias voltage, the backward transition (MIT) occurs immediately.

At bias voltages close to the IMT, the equivalent RMS AC voltage amplitude causing IMT is considerably smaller than the remaining DC voltage required for the IMT. Reciprocally, the shift in the IMT threshold voltage in presence of a high-frequency excitation is larger than the equivalent RMS AC voltage in the excitation. A similar behavior is observed when linking the RMS AC signal to an equivalent DC voltage in a nonlinear system, when operating RF MEMS capacitive switches biased near the pull-in voltage ${ }^{38}$.

\section{$\mathrm{VO}_{2}$ actuation through interrupted $\mathrm{CPW}$}

We have fabricated co-planar wave-guides with a slit in the middle of the signal line, positioned on a $\mathrm{VO}_{2}$ patch. Directly applying the mm-wave power on the small $\mathrm{VO}_{2}$ slit by the two VNA ports connected to the CPW, we try to minimize possible transmission losses through the simplest insertion of a $\mathrm{VO}_{2}$ patch at the exposed area, in order to extract the effect of high-frequency signals on $\mathrm{VO}_{2} \mathrm{DC}$ characteristics with the highest possible resolution. Using AC/DC decouplers, voltage-controlled $\mathrm{I}(\mathrm{V})$ current limited measurements were performed on the two terminals of the interrupted CPW. The configuration of the probes on the CPW under the microscope is shown in fig. 3(a).

Despite the relatively sharp IMT by voltage actuation, $\mathrm{VO}_{2}$ transition has a tail in resistance versus temperature characteristics for a few Celsius degrees, as well as an observable slope. We measured the $\mathrm{S}$ parameters at several temperatures from $25^{\circ} \mathrm{C}$ up to $90^{\circ} \mathrm{C}$, having $\mathrm{VO}_{2}$ half way of transition and above that. fig. $3(\mathrm{~b})$ shows the average magnitude of $\mathrm{S}$ parameters for the CPW over frequencies from $60 \mathrm{GHz}$ up to $0.11 \mathrm{THz}$ in intermediate states of $\mathrm{VO}_{2}$ from semiconducting to metallic. The evolution of the curves at higher temperatures is quite consistent with the gradual expansion of metallic domains in $\mathrm{VO}_{2}$ by temperature ${ }^{30}$.

We measured the static I-V characteristics of $\mathrm{VO}_{2}$ inside the $\mathrm{CPW}$ in presence of a wide-band signal ranging from above mm-wave $(60 \mathrm{GHz})$ up to $0.11 \mathrm{THz}$. The curves reveal a considerable shift in the static IMT critical voltage of $\mathrm{VO}_{2}$. At each power, 100 double-sweeps of voltage are recorded and plotted for each of the figs. 4(a) through $4(\mathrm{c})$, at room temperature, $40^{\circ}$, and $50^{\circ}$, respectively. The curve-splitting gets smaller at temperatures closer to $T_{I M T}$ as expected, besides the additional thermal fluctuations which reduce the resolution between the curves at different power levels. The extracted probability densities of the IMT threshold voltages are depicted in figs. 4(d) through (f) for each power level, showing a noticeable bimodal behavior. 


\section{Readout circuit}

The 2-terminal $\mathrm{VO}_{2}$ devices function as active elements in the astable multivibrator circuit of fig. 1(c) The output signal of the oscillator was recorded for several thousands of cycles at each power level. We notice that the average $\mathrm{V}_{\text {IMT }}$ threshold sensitivity to incident power is much higher than that of the average $\mathrm{V}_{\text {MIT }}$ threshold. Thus, we obtain a modulation of the hysteresis window width as a function of applied external RF power.

Any change in the IMT threshold voltage modulates the intersection of the $\mathrm{VO}_{2}$ device I-V characteristics with that of the transistor in a series configuration (fig. 1(d)). As long as the excursion of the operating point does not reach the triode region of the MOSFET, any change in hysteresis window width will translate into a proportional change in operating frequency, as shown in the fast fourier transform (FFT) spectra of fig. 5(a).

The histograms at each power level (figs. 5(d) to (i)) show bimodal distributions which can be related to the threshold voltage distributions of figs. 4(d) to (f) through the non-linear oscillator transfer function.

Based on Allan deviation calculations, we were able to identify four main noise behaviors. One related to thermal noise, leads to a Normal statistic and dominates for averaging times below $200 \mathrm{~ms}$. The lowest noise level around $200 \mathrm{~ms}$ is usually related to $1 / \mathrm{f}$ (Flicker) noise. The next rise in the distribution noise can be related to the nucleation Poissonian processes and dominates for longer time constants. Final bumps are mostly due to parasitic signals, inter-modulation, low-frequency interference, instabilities of supply voltage, etc. It then limits the potential averaging of successive frequency measurements to reduce their standard deviation. Both mechanisms combine to induce threshold fluctuations, act as precursors of a bifurcation, and appear to form the basis of the stochastic oscillating behavior.

\section{Detection capability}

The shift in the static IMT critical voltage in presence of a high-frequency signal, with respect to the baseline static curve (the one with no additional high-frequency power), is a criteria for the "response" of the $\mathrm{VO}_{2}$-based devices. Using a proper read-out circuit, we can exploit the sensing capability of such a response in mm-wave and $\mathrm{THz}$ applications. Responsivity, or $R_{V}$, defined here as the ratio of the shift in the required voltage for IMT to the radiation power from the emitting port, is plotted in fig. 6(a) versus the corresponding power. The responsivity in this plot is based on the nominal power from the source, while if we consider only the received power by the $\mathrm{VO}_{2}$ sensing area, the responsivity gets at least one order of magnitude higher.

We apply the same concept for responsivity in the frequency of oscillations, defined by the deviation in the central frequency of oscillations, divided by the corresponding power. (fig. 6(a)). The baseline is the frequency of oscillations when no external power is applied.

The NEP of a detector is theoretically defined as the ratio between the noise spectral density (NSD) and the responsivity. One source of noise in this detector is the resistive $\mathrm{VO}_{2}$ area responsible for Johnson-Nyquist noise, which depends on temperature and resistance by $\sqrt{4 k_{B} T R \Delta f}$, where $R$ is the resistance of the active detecting area and $\Delta f$ is the measurement single-sided bandwidth. Another source is the shot noise of the bias current. The root mean square (RMS) current fluctuations as a Poisson process has a magnitude of $\sqrt{2 q I_{d c} \Delta f}$, where $q$ is the charge of an electron and $I_{d c}$ is the bias current. In order to experimentally evaluate the noise in static measurements, we apply a constant voltage close to $V_{I M T}$ and record the fluctuations in the current over 100 seconds, and calculate the NEP down to $4 \mathrm{mHz}$ through the FFT of the signal fluctuations (fig. 6(b)). The maximum NEP among all the cases happens when the temperature goes high enough so that the degradation in responsivity dominates the decline in resistance, which explains the worst NEP in $\mathrm{CPW}$ devices at $50^{\circ} \mathrm{C}$. At room temperature, we consider the worst NEP at low frequencies, which includes collectively the effect of material drifts, thermal fluctuations, and other external noises regarding the setup. The upper bound of the NEP in this case is near $20 \mathrm{nW}$.

We also consider the intrinsic noise of the stochastic behavior in phase transition. The fitted standard deviation $(\sigma)$ of $\mathrm{V}_{\text {IMT }}$ in CPW devices at room temperature, which is $2.77 \mathrm{mV}$ when no power applied, implies the limit of detection (LoD). The LoD can be estimated by $3 \sigma / R_{V}$ at lowest actuation level, which gives the value of $84 n W$. However, the standard deviation for the frequency of oscillations according to Allan method ${ }^{39}$ goes down to $1.3 \mathrm{~Hz}$ for averaging times around $200 \mathrm{~ms}$ when no power or very low power is applied (fig. 6(c)). In this case the equivalent noise power will be $58 \mathrm{nW}$.

We note that the calculated noise here is the "optical equivalent noise", as the responsivity that we used here is based on the emitted power and not the absorbed power. Thus, if one excludes the coupling loss from the responsivity and calculates the "electrical NEP", the value will be much smaller, only representing the intrinsic performance limits of the device. 


\section{Discussion}

The demonstration room-temperature actuation of $\mathrm{VO}_{2}$ by mm-wave and $\mathrm{THz}$ applied power proves the opportunity of designing and manufacturing efficient sensor devices based on the phase change phenomena. We first improved the sensitivity of $\mathrm{VO}_{2}$ to low-power radiation by means of a DC-bias-assisted setting inside an antenna and investigated its properties in a CPW configuration.

We demonstrated the density-driven IMT by radiation in $\mathrm{VO}_{2}$ through splitting the $\mathrm{S}$ parameters in a twocoupled-antenna setup, where $\mathrm{VO}_{2}$ is embedded in one of them. Furthermore, the effect of external radiation on the static I-V characteristics of $\mathrm{VO}_{2}$ is shown as a shift in the threshold voltage required to induce IMT. This effect can form the principle of detection in $\mathrm{mm}$-wave and $\mathrm{THz}$ detectors. We reported the extent of this shift normalized by the radiation power as the nominal responsivity of the device in fig. 6(a). This is while the actual responsivity as a function of absorbed power is at least 37 times higher than the values reported in this figure for the case of the antennas, and more than three times higher in the case of CPWs. With this definition, the equivalent noise power will also get lower proportionally.

Concerning the nonlinear responsivity in fig. 6(a), the extent of the shift at the lowest incident power compared to the shift due to higher levels of power, confirms that the sensor shows a higher responsivity to smaller triggers. The equivalent RMS AC voltage is smaller than the actual DC shift that it causes. Such behavior is usually found in avalanche phenomena, and here it can relate to the lattice instabilities driving the phase transition at a proper bias point. Hence, by device scaling and bias point engineering, this design will be promising for a super-sensitive, high-responsivity detector.

Regarding sensor applications, the above-described method and devices appear to be applicable over a relatively wide range in temperature from cooled conditions (the IMT voltage is always assisted by a calibrated DC voltage) up to around $40^{\circ} \mathrm{C}$. The experimental figures of merit of the power sensor proposed here are comparable or even outperform other works that report power sensing based on graphene, Silicon FETs, and some other $\mathrm{VO}_{2}$-based devices as summarized in Table 1.

Our results suggest that for the design and performance optimization of a spiking $\mathrm{VO}_{2}$ sensor, it is important to: (i) design low-voltage actuation of structures (similarly with low-voltage-actuated MEMS with soft springs), therefore further miniaturization of device length and field concentrators are expected to help to further enhance the sensing characteristics, (ii) improve the high-frequency power coupling into the 2-terminal $\mathrm{VO}_{2}$ device by better antennas and CPW design and reduce the parasitic effects, (iii) consider operating the device near the switching point and combine with the use of a local heater.

\begin{tabular}{|l|l|l|l|l|}
\hline Detector type & $\mathrm{R}_{\mathrm{V}}$ & NEP $\left[\mathrm{nW} / \mathrm{Hz}^{0.5}\right]$ & Frequency $(\mathrm{THz})$ & Reference \\
\hline Graphene-based & $20-600 \mathrm{~V} / \mathrm{W}$ & $0.1-1$ & $0.01-0.8$ & $40-47$ \\
\hline Si FET & $10-5000 \mathrm{~V} / \mathrm{W}$ & $0.01-199$ & $0.1-1.63$ & $48-53$ \\
\hline${ }^{*} \mathrm{VO}_{2}$-based & $21.28 \mathrm{GHz} / \mathrm{W}$ & NA & Optical & 54 \\
\hline This work & DC: $76 \mathrm{kV} / \mathrm{W}, \mathrm{AC}: 66.3 \mathrm{GHz} / \mathrm{W}$ & 20 & $0.01-0.22$ & \\
\hline
\end{tabular}

Table 1. Comparison of detector performances in different technologies

\section{Methods}

\section{$\mathrm{VO}_{2}$ deposition}

The thin film deposition is done on a $525 \mu \mathrm{m}$-thick high resistivity float zone (HRFZ) Si wafer $(>10000 \Omega . \mathrm{cm})$. The substrate was then passivated by growth of $2 \mu \mathrm{m} \mathrm{SiO}$ thermal wet oxide. The 100-nm-thick polycrystalline $\mathrm{VO}_{2}$ film was synthesized on this substrate through a pulsed laser deposition (PLD) system using a $\mathrm{V}_{2} \mathrm{O}_{5}$ target, in high-vaccum conditions, in a chamber with pressure of $0.01 \mathrm{mbar}$ and Oxygen flow of $1 \mathrm{sccm}$. The laser energy was $400 \mathrm{~mJ}$ with a pulse frequency of $20 \mathrm{~Hz}$. The substrate temperature was kept at $400^{\circ} \mathrm{C}$ during the deposition, and the process was followed by post annealing at $475^{\circ} \mathrm{C}$.

\section{Device fabrication}

The $\mathrm{VO}_{2}$ film was patterned on a 100-nm-thick $\mathrm{SiO}_{2}$ layer, using photolithography on negative tone $\mathrm{AZ}$ nLof 2020 resist followed by wet etching in diluted $\mathrm{Cr}$ etch solution. For the second layer, $15 \mathrm{~nm} \mathrm{Cr}$ was sputtered on the patterned substrate as an interface layer and the process was followed by the sputtering of $150 \mathrm{~nm} \mathrm{Au}$. The 
connections and the 2-terminal devices were completed by dry etching of the sputtered metal using a broad beam of Argon ions in an ion-beam-etcher. A more detailed process flow is presented in the supplementary materials.

\section{Electrical measurements}

High and low frequency measurements were all performed on a Cascade Summit probe station. Mm-wave measurement incorporated broadband VNA system with MPI GSG 220-GHz infinity probes, each of them connected to a port from ANRITSU Vector Star VNA through frequency multiplier modules based on nonlinear transmission line (NLTL).

DC measurements were planned on a multi-cycle mode using Keithley semiconductor parameter analyzer. Each series of the DC measurements were performed at least a hundred cycles in a row to avoid transient results for the static characteristics of $\mathrm{VO}_{2}$.

\section{References}

1. Morin, F. J. Oxides which show a metal-to-insulator transition at the neel temperature. Phys. Rev. Lett. 3, 34-36, DOI: 10.1103/PhysRevLett.3.34 (1959).

2. Berglund, C. \& Guggenheim, H. Electronic properties of v o 2 near the semiconductor-metal transition. Phys. Rev. 185, 1022 (1969).

3. Pellegrino, L. et al. Multistate memory devices based on free-standing vo2/tio2 microstructures driven by joule self-heating. Adv. Mater. 24, 2929-2934 (2012).

4. Cabrera, R., Merced, E. \& Sepúlveda, N. A micro-electro-mechanical memory based on the structural phase transition of vo2. physica status solidi (a) 210, 1704-1711 (2013).

5. Mazza, G., Amaricci, A., Capone, M. \& Fabrizio, M. Field-driven mott gap collapse and resistive switch in correlated insulators. Phys. review letters 117, 176401 (2016).

6. Kalcheim, Y. et al. Robust coupling between structural and electronic transitions in a mott material. Phys. review letters 122, 057601 (2019).

7. Miller, K. J., Hallman, K. A., Haglund, R. F. \& Weiss, S. M. Silicon waveguide optical switch with embedded phase change material. Opt. express 25, 26527-26536 (2017).

8. Fan, L. et al. Infrared response and optoelectronic memory device fabrication based on epitaxial vo2 film. ACS applied materials $\mathcal{E}$ interfaces 8, 32971-32977 (2016).

9. Koledov, V. et al. Interaction of electromagnetic waves with vo2 nanoparticles and films in optical and millimetre wave ranges: Prospective for nano-photonics, nano-antennas, and sensors. In Journal of Physics: Conference Series, vol. 1092, 012108 (2018).

10. Kim, B.-J. et al. Temperature dependence of the first-order metal-insulator transition in vo 2 and programmable critical temperature sensor. Appl. physics letters 90, 023515 (2007).

11. Benkahoul, M. et al. Thermochromic vo2 film deposited on al with tunable thermal emissivity for space applications. Sol. Energy Mater. Sol. Cells 95, 3504-3508 (2011).

12. Lei, L. et al. Tunable and scalable broadband metamaterial absorber involving vo2-based phase transition. Photon. Res. 7, 734-741, DOI: 10.1364/PRJ.7.000734 (2019).

13. Brockman, J. S. et al. Subnanosecond incubation times for electric-field-induced metallization of a correlated electron oxide. Nat. Nanotechnol. 9, 453-458 (2014).

14. Lappalainen, J., Mizsei, J. \& Huotari, M. Neuromorphic thermal-electric circuits based on phase-change vo2 thin-film memristor elements. J. Appl. Phys. 125, 044501 (2019).

15. Corti, E. et al. Scaled resistively-coupled vo2 oscillators for neuromorphic computing. Solid-State Electron. 168, 107729 (2020).

16. Ghann, W. \& Uddin, J. Terahertz (thz) spectroscopy: A cutting edge technology. Terahertz Spectrosc. Cut. Edge Technol. (2017).

17. Kulesa, C. Terahertz spectroscopy for astronomy: From comets to cosmology. IEEE Transactions on Terahertz Sci. Technol. 1, 232-240, DOI: 10.1109/TTHZ.2011.2159648 (2011).

18. Choudhury, B., Menon, A. \& Jha, R. M. Active Terahertz Metamaterial for Biomedical Applications, 1-41 (Springer Singapore, Singapore, 2016). 
19. Mittleman, D. M. Twenty years of terahertz imaging. Opt. Express 26, 9417-9431, DOI: 10.1364/OE.26.009417 (2018).

20. Corsi, C. \& Sizov, F. THz and security applications: detectors, sources and associated electronics for THz applications (Springer, 2014).

21. Koenig, S. et al. Wireless sub-THz communication system with high data rate. Nat. Photonics 7, 977-981, DOI: 10.1038/nphoton.2013.275 (2013).

22. Duchene, J., Terraillon, M., Pailly, P. \& Adam, G. Filamentary Conduction in VO2 Coplanar Thin-Film Devices. Appl. Phys. Lett. 19, 115-117, DOI: 10.1063/1.1653835 (1971). Publisher: American Institute of Physics.

23. Lee, J., Ortolani, M., Schade, U., Chang, Y. \& Noh, T. Microspectroscopic detection of local conducting areas generated by electric-pulse-induced phase transition in vo 2 films. Appl. Phys. Lett. 91, 133509 (2007).

24. Lee, J. et al. Electric-pulse-induced local conducting area and joule heating effect in vo2/al2o3 films. Infrared physics \& technology 51, 443-445 (2008).

25. Okimura, K., Ezreena, N., Sasakawa, Y. \& Sakai, J. Electric-field-induced multistep resistance switching in planar VO2/c-al2o3structure. Jpn. J. Appl. Phys. 48, 065003, DOI: 10.1143/jjap.48.065003 (2009).

26. Pevtsov, A. B. et al. Evidence of field-induced nucleation switching in opal: $\mathrm{Vo}_{2}$ composites and $\mathrm{vo}_{2}$ films. Phys. Rev. B 85, 024110, DOI: 10.1103/PhysRevB.85.024110 (2012).

27. Gopalakrishnan, G., Ruzmetov, D. \& Ramanathan, S. On the triggering mechanism for the metal-insulator transition in thin film vo 2 devices: electric field versus thermal effects. J. materials science 44, 5345-5353 (2009).

28. Diener, P. et al. How a dc electric field drives mott insulators out of equilibrium. Phys. Rev. Lett. 121, 016601, DOI: 10.1103/PhysRevLett.121.016601 (2018).

29. Stoliar, P. et al. Nonthermal and purely electronic resistive switching in a mott memory. Phys. Rev. B 90, 045146 (2014).

30. Yang, Z., Ko, C. \& Ramanathan, S. Oxide electronics utilizing ultrafast metal-insulator transitions. Annu. Rev. Mater. Res. 41, 337-367 (2011).

31. Kalcheim, Y. et al. Non-thermal resistive switching in mott insulator nanowires. Nat. Commun. 11, 1-9 (2020).

32. Bohaichuk, S. M. et al. Localized triggering of the insulator-metal transition in vo2 using a single carbon nanotube. ACS nano 13, 11070-11077 (2019).

33. Mayer, B. et al. Tunneling breakdown of a strongly correlated insulating state in $\mathrm{v}$ o 2 induced by intense multiterahertz excitation. Phys. Rev. B 91, 235113 (2015).

34. Wong, H. M. et al. Broadband, integrated, micron-scale, all-optical si3n4/vo2 modulators with pj switching energy. ACS Photonics 6, 2734-2740 (2019).

35. Madaras, S. E. et al. Dynamics of the blue pump-induced ultrafast insulator-to-metal transition and relaxation in vo 2/tio 2 and vo 2/tio 2: Nb thin films. Opt. Mater. Express 10, 1393-1404 (2020).

36. Tao, Z. et al. The nature of photoinduced phase transition and metastable states in vanadium dioxide. Sci. reports 6, 1-10 (2016).

37. Tadjer, M. J. et al. Temperature and electric field induced metal-insulator transition in atomic layer deposited vo2 thin films. Solid-State Electron. 136, 30-35 (2017).

38. Vitale, W. A., Fernández-Bolaños, M., Bazigos, A., Dehollain, C. \& Ionescu, A. M. Rf mems power sensors for ultra-low power wake-up circuit applications. In 2013 Proceedings of the European Solid-State Device Research Conference (ESSDERC), 288-291, DOI: 10.1109/ESSDERC.2013.6818875 (2013).

39. Jerath, K., Brennan, S. \& Lagoa, C. Bridging the gap between sensor noise modeling and sensor characterization. Measurement 116, 350-366 (2018).

40. Viti, L., Purdie, D. G., Lombardo, A., Ferrari, A. C. \& Vitiello, M. S. Hbn-encapsulated, graphene-based, room-temperature terahertz receivers, with high speed and low noise. Nano Lett. 20, 3169-3177, DOI: 10.1021/acs.nanolett.9b05207 (2020). PMID: 32301617, https:/ / doi.org/10.1021/acs.nanolett.9b05207.

41. El Fatimy, A. et al. Ultra-broadband photodetectors based on epitaxial graphene quantum dots. Nanophotonics 7, 735-740 (2018). 
42. Viti, L., Purdie, D. G., Lombardo, A., Ferrari, A. C. \& Vitiello, M. S. Hbn-encapsulated, graphene-based, room-temperature terahertz receivers, with high speed and low noise. Nano Lett. 20, 3169-3177 (2020).

43. GK, R. M., Deshmukh, P., Prabhu, S. \& Basu, P. K. Antenna coupled graphene-fet as ultra-sensitive room temperature broadband thz detector. AIP Adv. 8, 125122 (2018).

44. Bandurin, D. et al. Dual origin of room temperature sub-terahertz photoresponse in graphene field effect transistors. Appl. Phys. Lett. 112, 141101 (2018).

45. Generalov, A. A., Andersson, M. A., Yang, X., Vorobiev, A. \& Stake, J. A 400-ghz graphene fet detector. IEEE Transactions on Terahertz Sci. Technol. 7, 614-616 (2017).

46. Andersson, M. A. \& Stake, J. An accurate empirical model based on volterra series for fet power detectors. IEEE Transactions on Microw. Theory Tech. 64, 1431-1441 (2016).

47. Tong, J. et al. Asymmetric 2-terminal graphene detector for broadband radiofrequency heterodyne-and self-mixing. Nano letters 18, 3516-3522 (2018).

48. Khan, M. I. W. et al. Nonlinear analysis of nonresonant thz response of mosfet and implementation of a high-responsivity cross-coupled thz detector. IEEE Transactions on Terahertz Sci. Technol. 8, 108-120 (2018).

49. Deng, X., Simanullang, M. \& Kawano, Y. Ge-core/a-si-shell nanowire-based field-effect transistor for sensitive terahertz detection. In Photonics, vol. 5, 13 (Multidisciplinary Digital Publishing Institute, 2018).

50. Ponomarev, D. et al. Sub-terahertz fet detector with self-assembled sn-nanothreads. J. Phys. D: Appl. Phys. 53, 075102 (2019).

51. Kopyt, P., Salski, B., Pacewicz, A., Zagrajek, P. \& Marczewski, J. Measurements of the responsivity of fet-based detectors of sub-thz radiation. Opto-Electronics Rev. 27, 123-129 (2019).

52. Ikamas, K., Nevinskas, I., Krotkus, A. \& Lisauskas, A. Silicon field effect transistor as the nonlinear detector for terahertz autocorellators. Sensors 18, 3735 (2018).

53. Qayyum, S. \& Negra, R. $0.8 \mathrm{mw}, 0.1-110 \mathrm{ghz}$ rf power detector with $6 \mathrm{ghz}$ video bandwidth for multigigabit software defined radios. In 2017 IEEE MTT-S International Microwave Symposium (IMS), 1722-1725 (IEEE, 2017).

54. Kim, B.-J., Seo, G., Choi, J., Kim, H.-T. \& Lee, Y. W. Laser-assisted control of electrical oscillation in vo2 thin films grown by pulsed laser deposition. Jpn. J. Appl. Phys. 51, 107302 (2012).

\section{Acknowledgements}

This project has been funded by the European Research Council (ERC) via the Advanced ERC Grant Millitech, having as Principal Investigator professor Mihai Adrian Ionescu.

\section{Author contributions statement}

A.M.I. and F.Q. developed the device principle. F.Q. optimized the $\mathrm{VO}_{2}$ thin film deposition recipe and fabricated the devices. F.Q. conducted static measurements on the sensor. F.Q. and T.R. worked on the oscillator circuit experiment. D.F. analyzed the bimodal distributions and the Allan deviation. F.Q., M.B. and J.L. conducted the high-frequency measurements. F.Q. and A.M.I. wrote the manuscript.

\section{Additional information}

Supplementary materials are submitted together with the manuscript. 


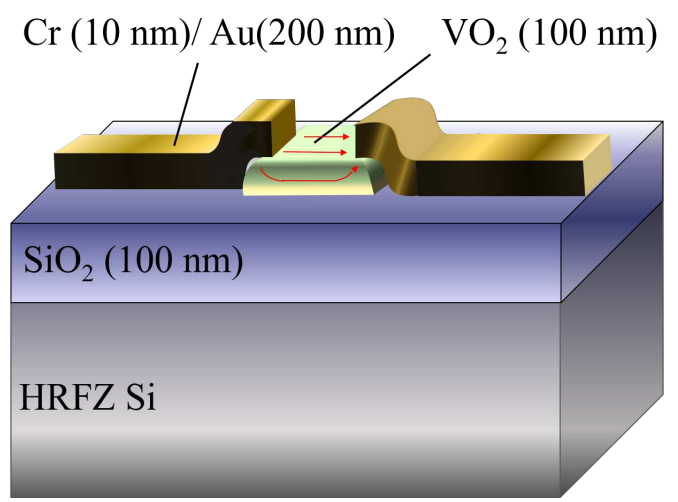

(a)

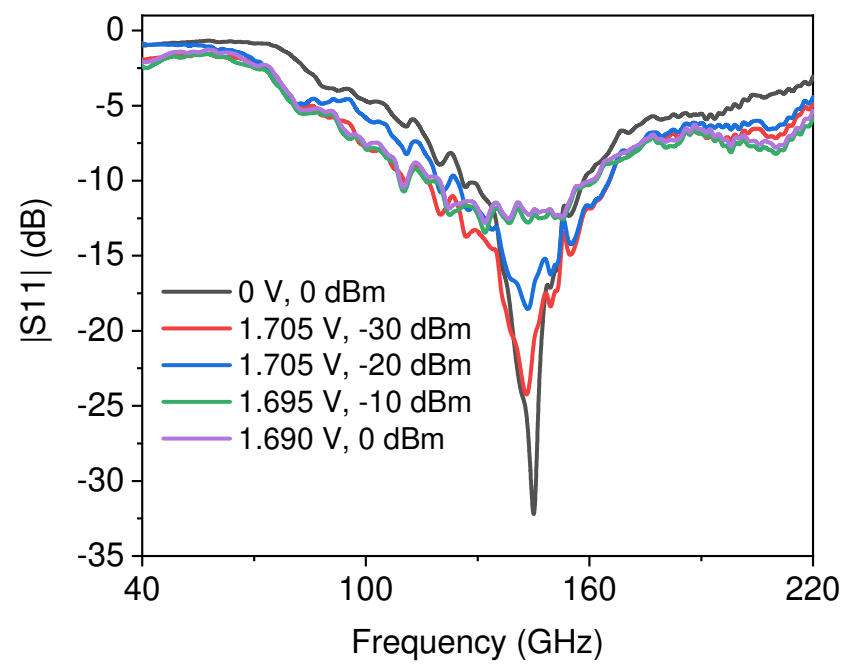

(c)

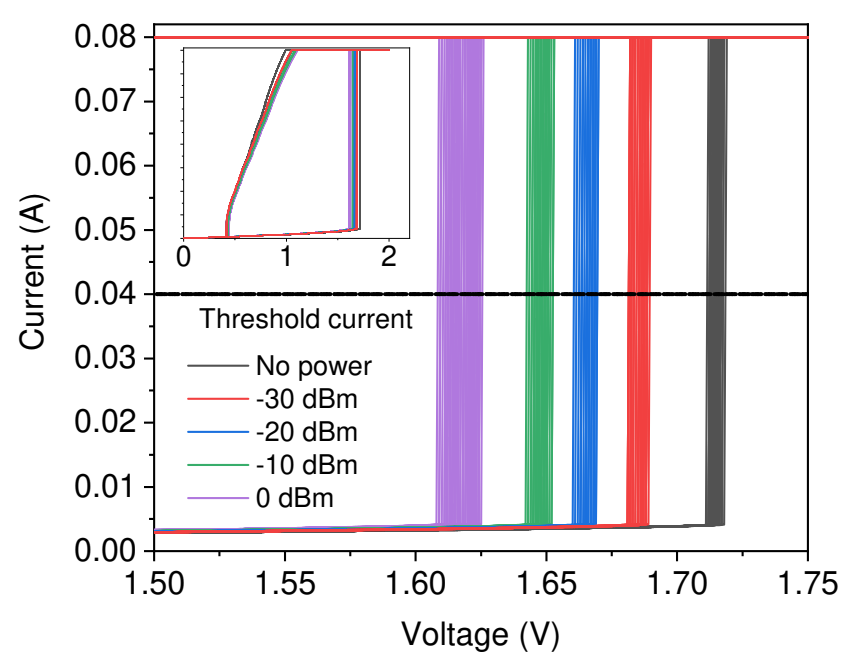

(e)

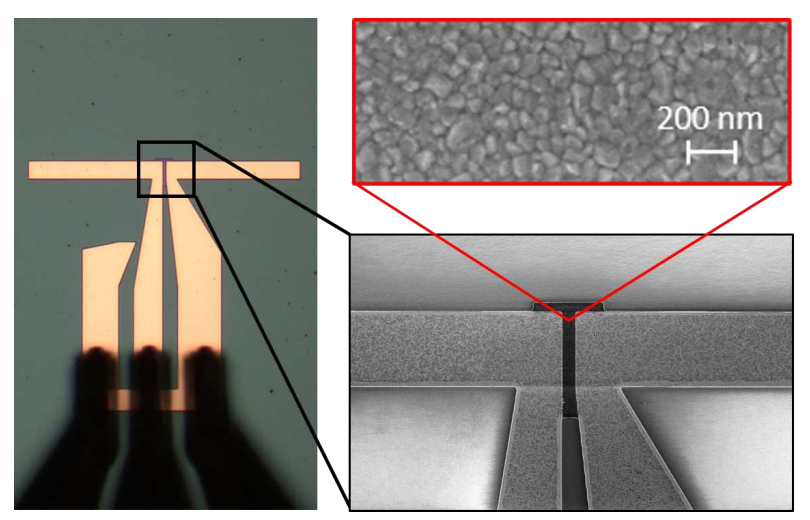

(b)

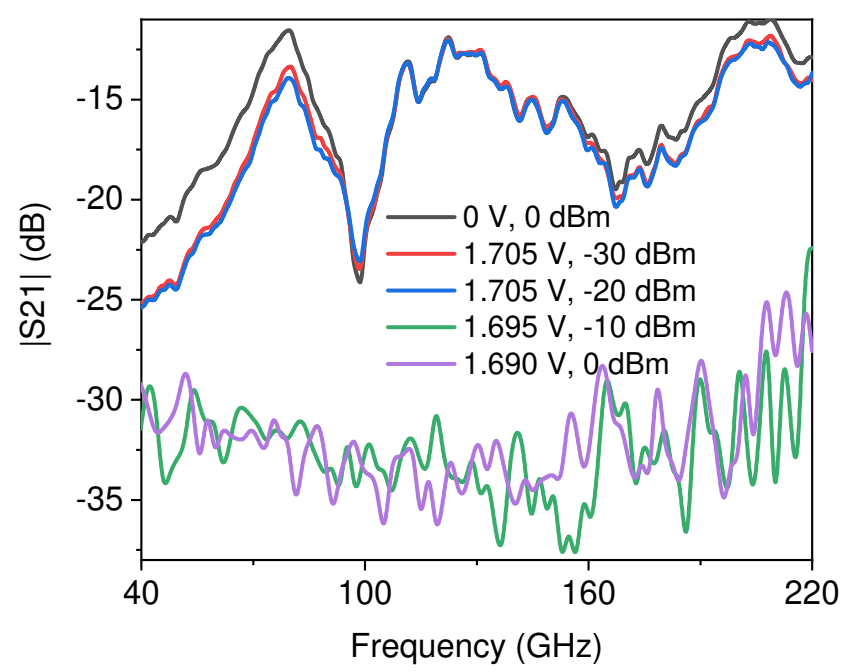

(d)

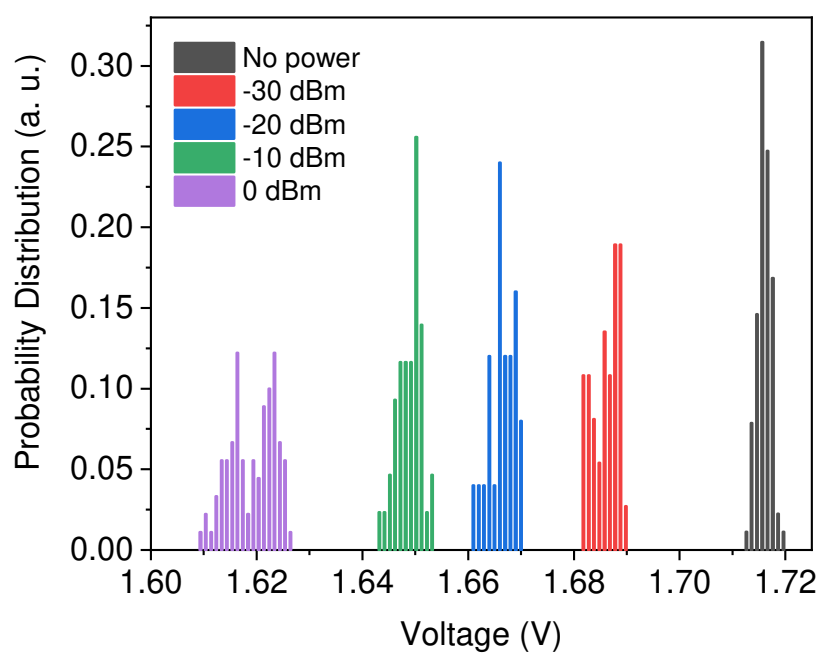

(f)

Figure 2. Coupled dipole antennas. (a) cross section of the central part in the receiver antenna (Note: the emitting antenna has no $\mathrm{VO}_{2}$ area), (b) microscopic image of the receiver antenna along with the zoomed SEM image, (c) $\left|S_{11}\right|$ for the emitting port for different bias voltages: impedance mismatch trend representing the transition in $\mathrm{VO}_{2}$ shunting the receiver, (d) $\left|S_{21}\right|$ for the coupled antennas at different bias voltages: splitting curves in "on" and "off" states according to the impedance transition, (e) multi-cycle measurement of I-V characteristics for $\mathrm{VO}_{2}$ in the receiving antenna, showing the shift in the IMT threshold voltage according to the external power. Inset: an overview of the whole characteristics, (f) probability density of IMT threshold voltages at each power 


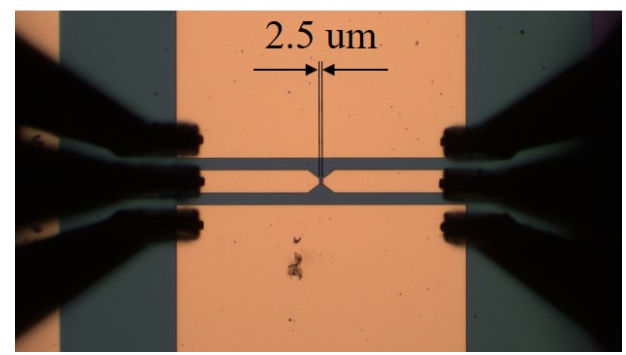

(a)

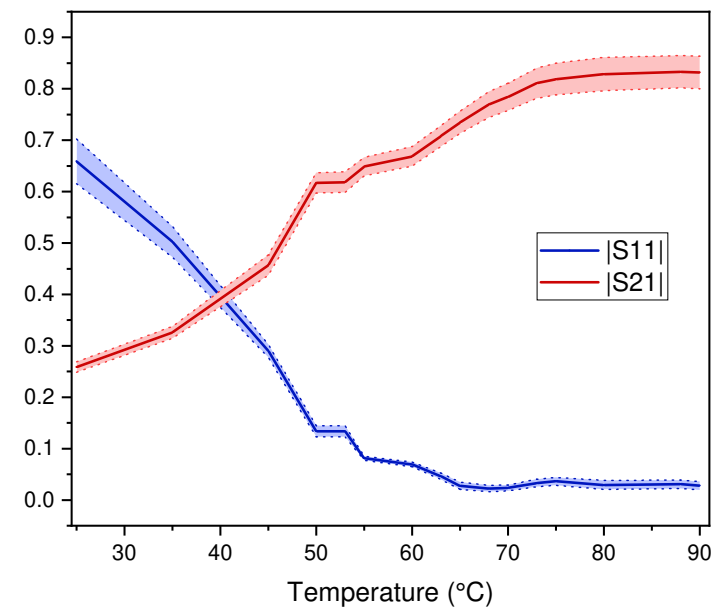

(b)

Figure 3. interrupted $\mathrm{CPW}$ by VO2. (a) under the microscope, (b) S parameters magnitude between the two ports across the $\mathrm{CPW}$ vs. temperature 


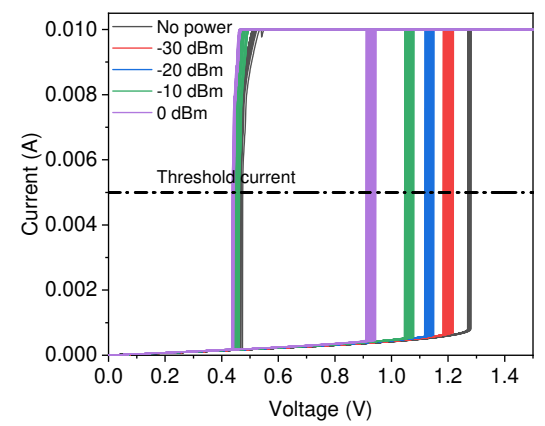

(a)

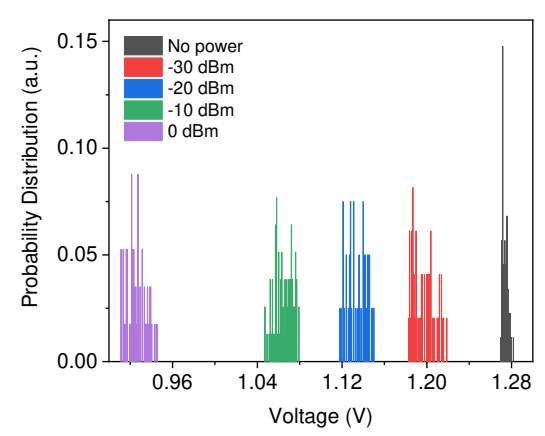

(d)

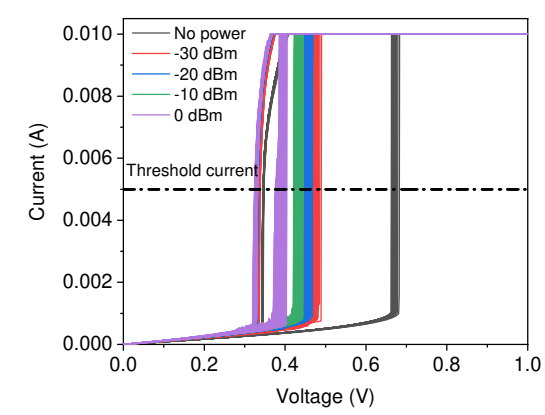

(b)

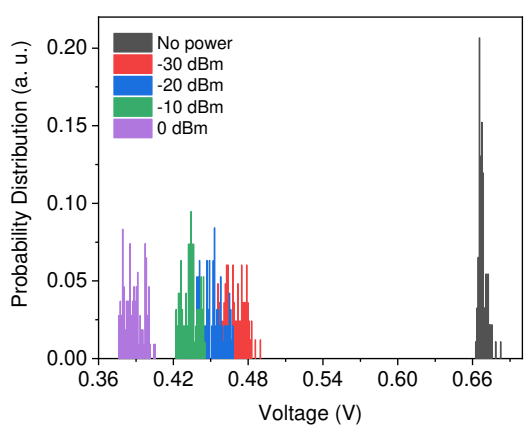

(e)

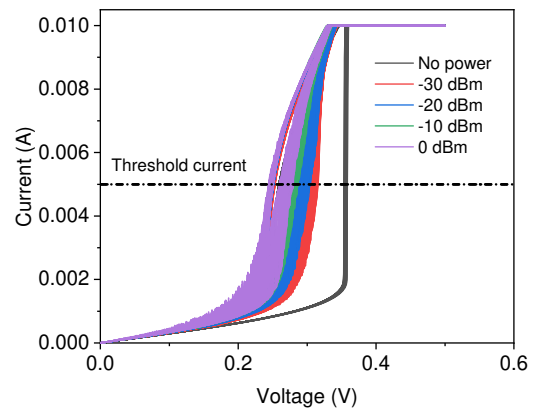

(c)

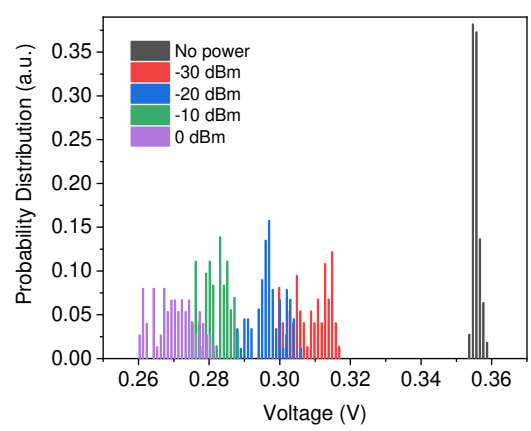

(f)

Figure 4. (a) - (c) static $\mathrm{I}-\mathrm{V}$ characteristics of $\mathrm{VO}_{2}$ with respect to different levels of external power, at (a) room temperature, (b) $40^{\circ} \mathrm{C}$, and (c) $50^{\circ} \mathrm{C}$. (d) - (f) probability distribution of IMT threshold voltage, at (d) room temperature, (e) $40^{\circ} \mathrm{C}$, and (f) $50^{\circ} \mathrm{C}$. Elevated temperatures make major shifts in the threshold voltage, while the effect of the external power is maintained in all cases. The temperature role is mainly on the resolution between the curves at different power levels. 


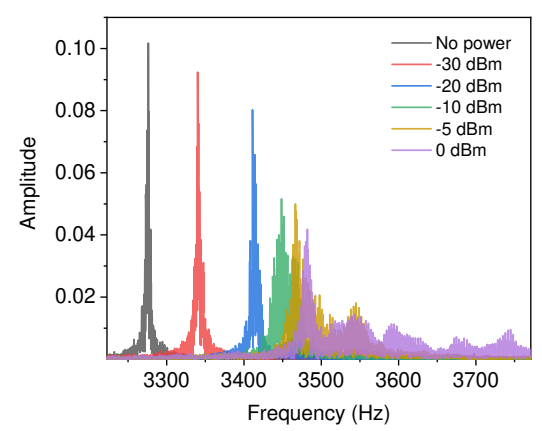

(a)

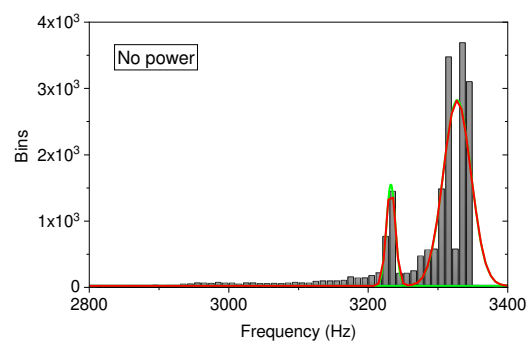

(d)

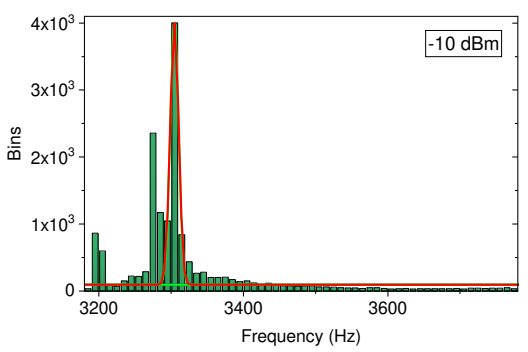

(g)

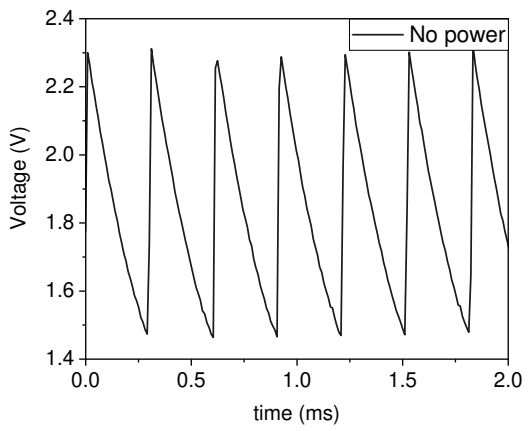

(b)

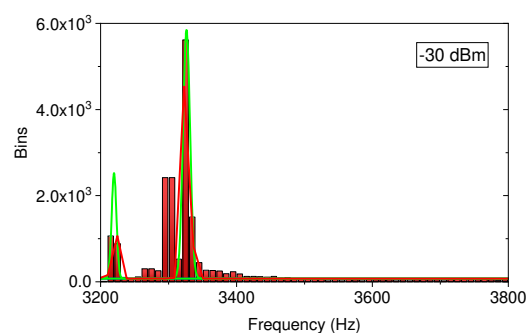

(e)

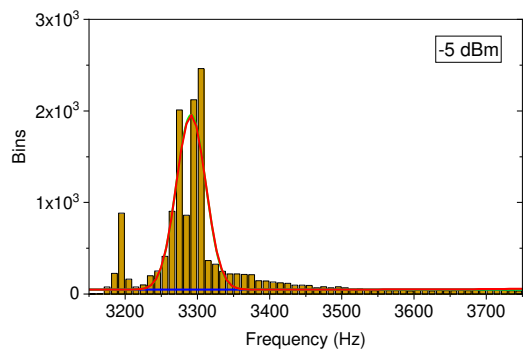

(h)

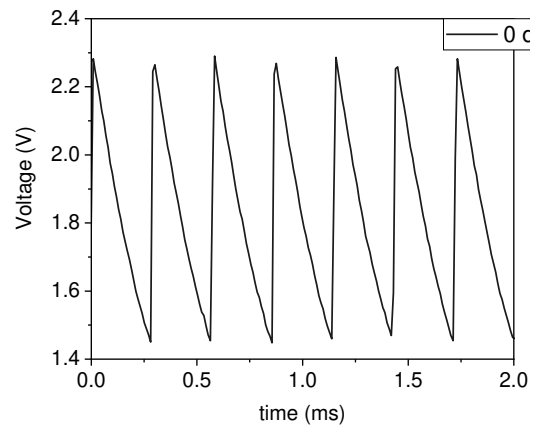

(c)

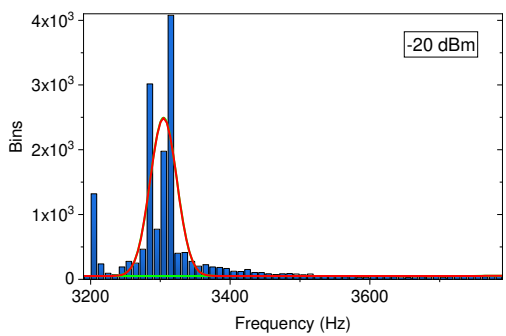

(f)

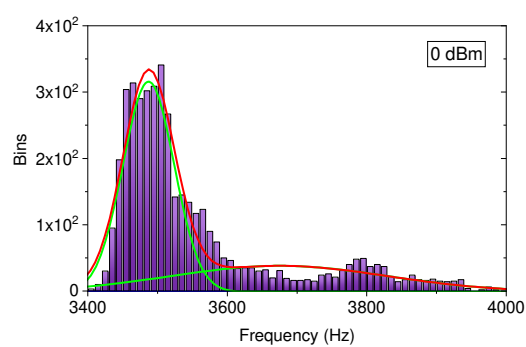

(i)

Figure 5. Frequency of oscillations based on applied power (a) FFT of output waveforms with respect to the applied power, (b) and (c) time domain waveforms for "no power" and "0 dBm" exposure, (d)-(i) distributions for the frequency of oscillations based on the emitted power from the first port. 


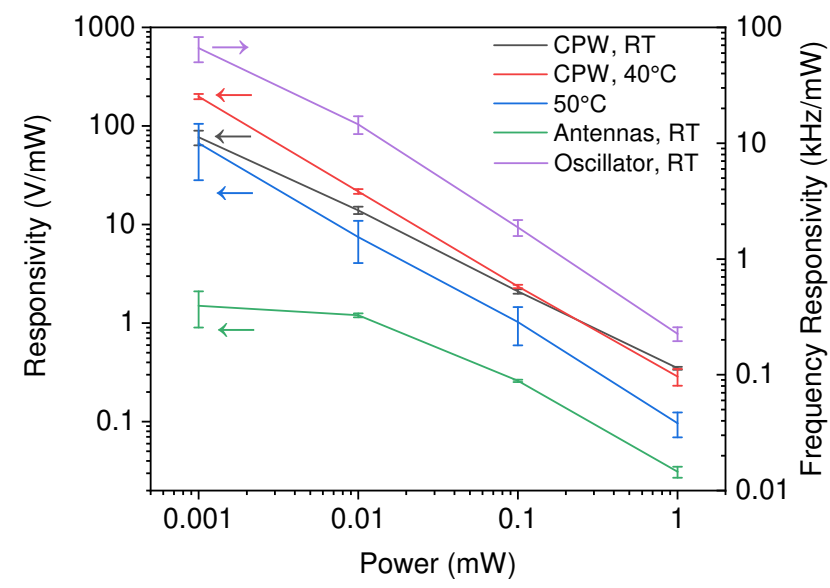

(a)

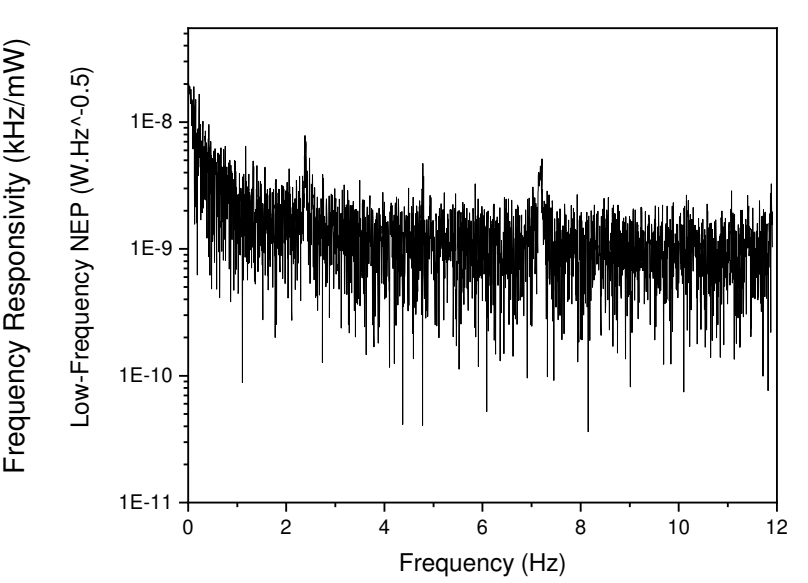

(b)

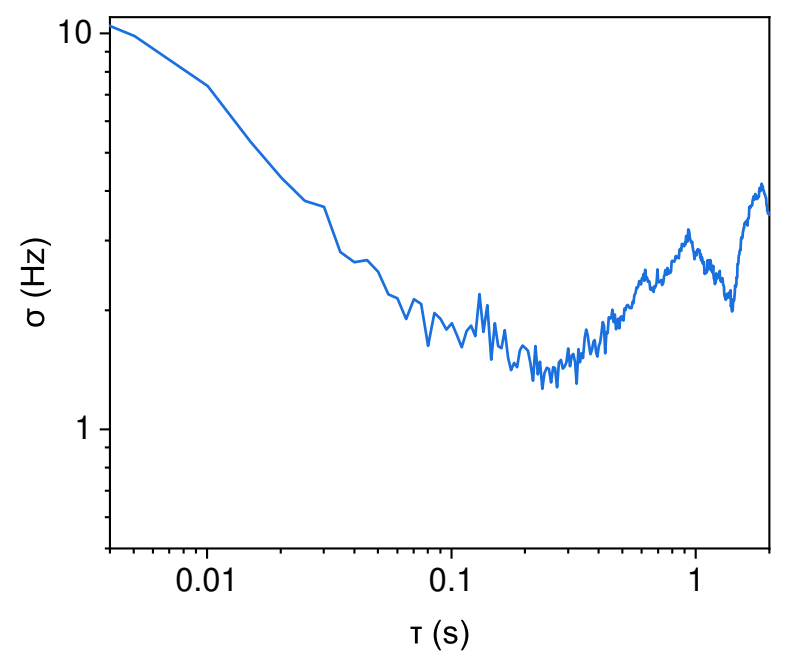

(c)

Figure 6. figure of merit for the proposed $\mathrm{VO}_{2}$-based sensor (a) $\mathrm{DC}$ and frequency responsivity due to the shift in the IMT threshold voltage for the mentioned configurations based on the emitted power from the first port, (b) Noise equivalent power of the CPW devices at very low frequencies, (c) Allan deviation calculation on the frequency vs time measurements of the oscillator over a long duration of time, no external power applied 


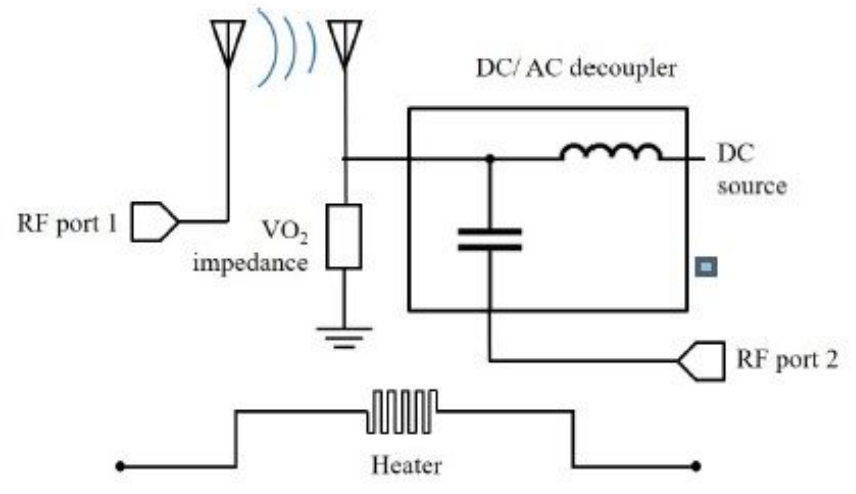

(a)

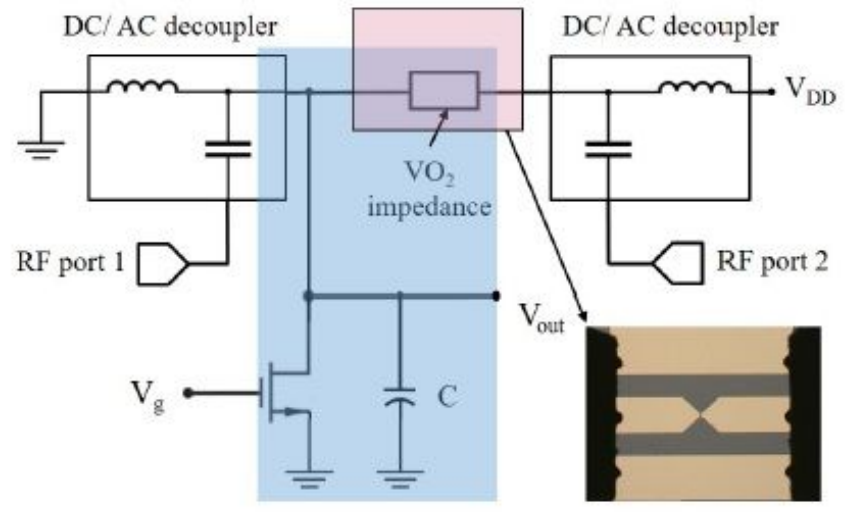

(c)

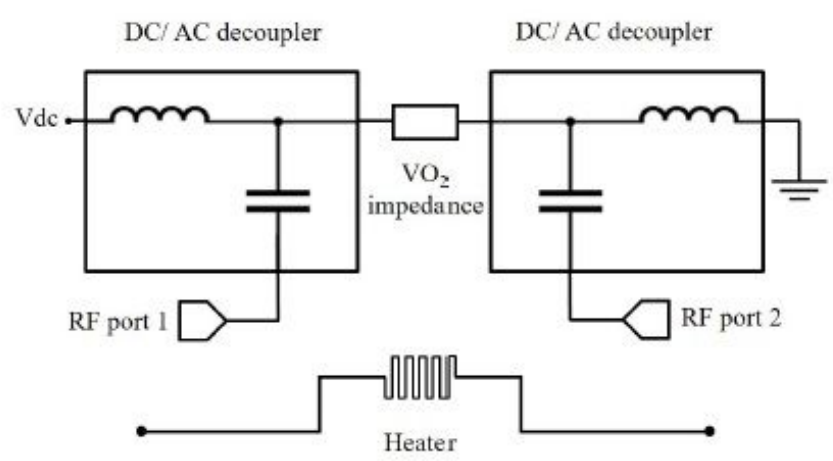

(b)

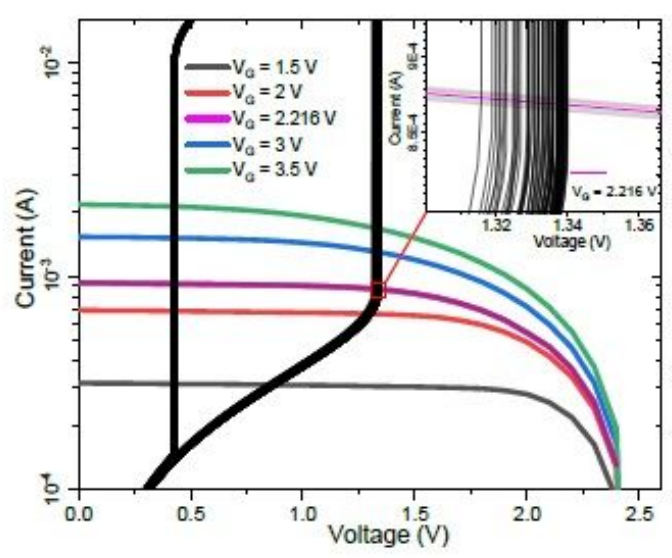

(d)

\section{Figure 1}

Depiction of the mm-wave detection and experimental setup used in this work. (a) VO2 in coupled antennas case, (b) VO2 in interrupted CPW case, (c) astable circuit using a 2-terminal VO2 device in interrupted CPW in series with a MOSFET, (d) the initial operation point of the oscillating sensor is set by the intersection of the static characteristics of VO2 and NMOS transistor at specific points supporting astability. Inset: the operational bias points and multiple cycles of experiments suggesting nondeterministic behavior. 


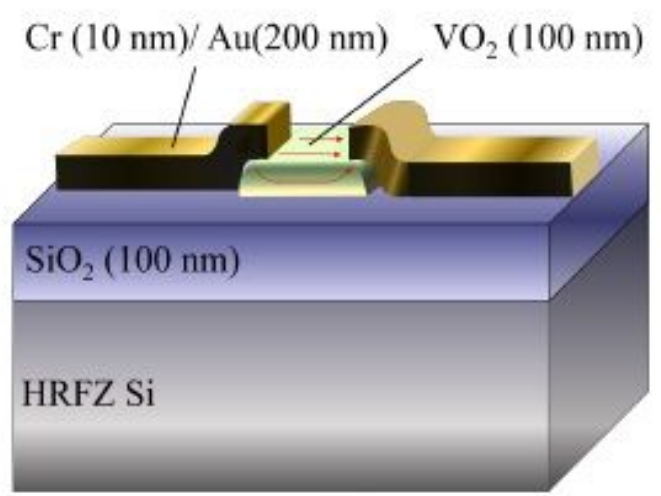

(a)

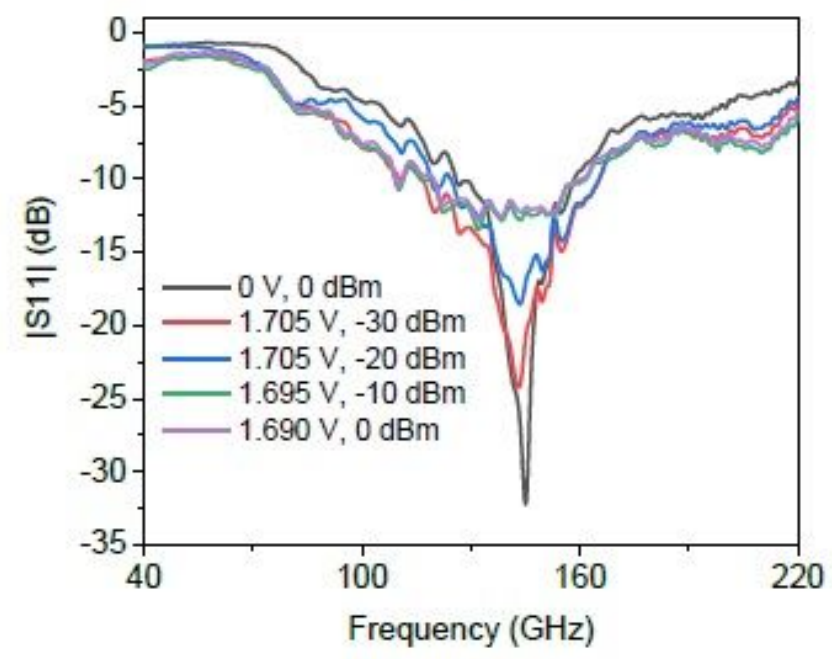

(c)

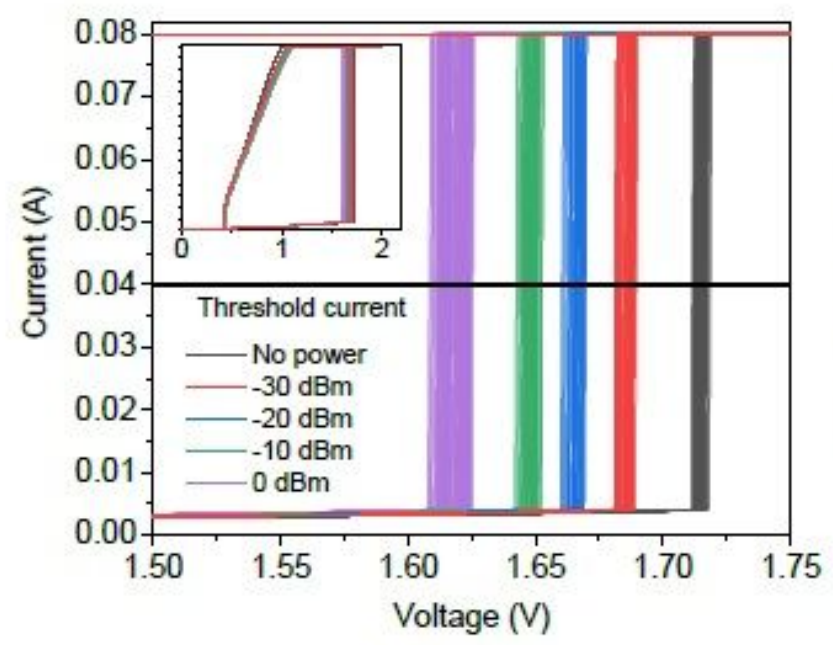

(e)

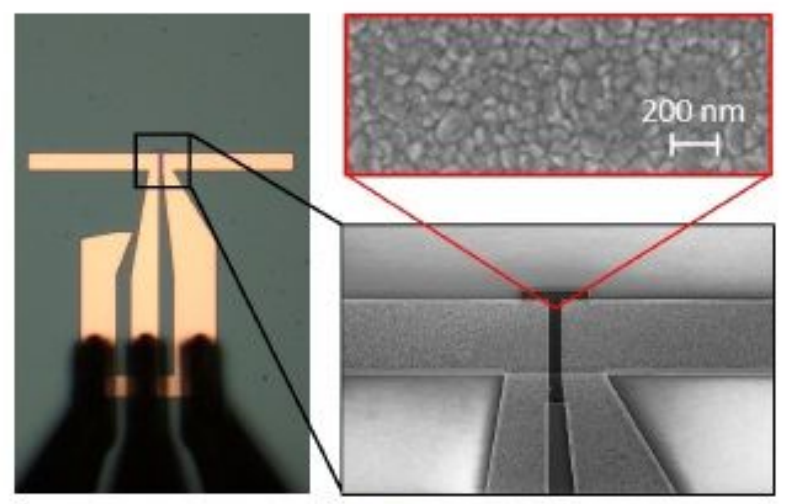

(b)

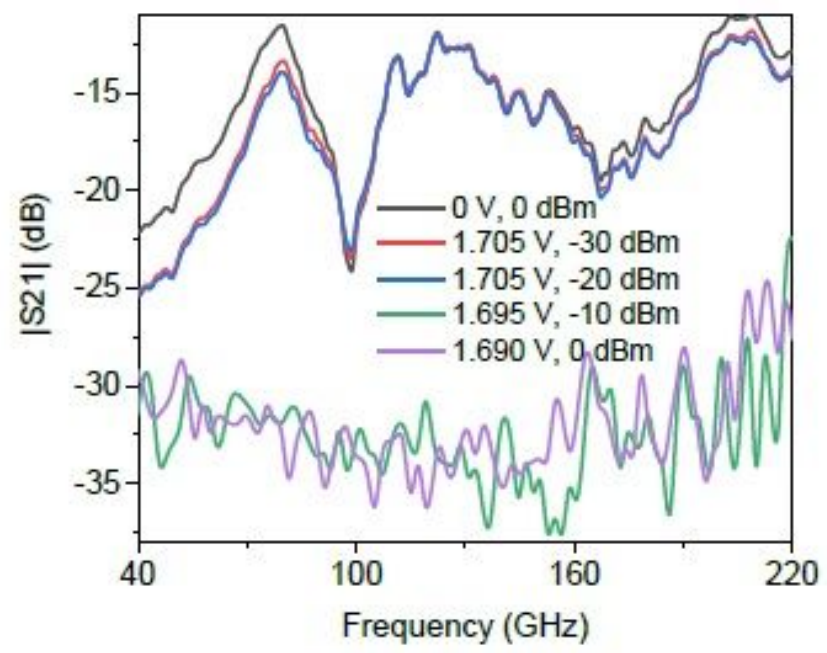

(d)

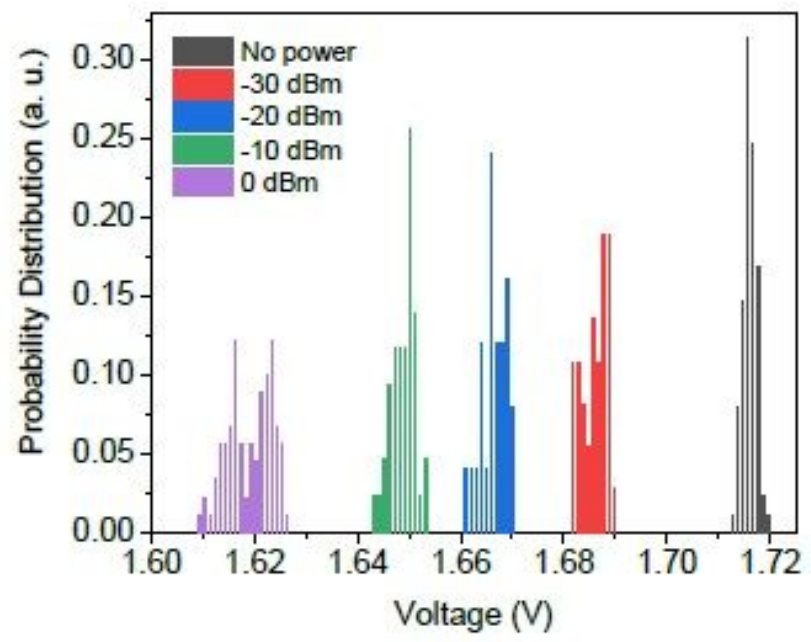

(f)

\section{Figure 2}

Coupled dipole antennas. (a) cross section of the central part in the receiver antenna (Note: the emitting antenna has no VO2 area), (b) microscopic image of the receiver antenna along with the zoomed SEM image, (c) jS11 j for the emitting port for different bias voltages: impedance mismatch trend representing the transition in VO2 shunting the receiver, (d) jS21 j for the coupled antennas at different bias voltages: splitting curves in "on" and "off" states according to the impedance transition, (e) multi-cycle 
measurement of I-V characteristics for VO2 in the receiving antenna, showing the shift in the IMT threshold voltage according to the external power. Inset: an overview of the whole characteristics, $(f)$ probability density of IMT threshold voltages at each power

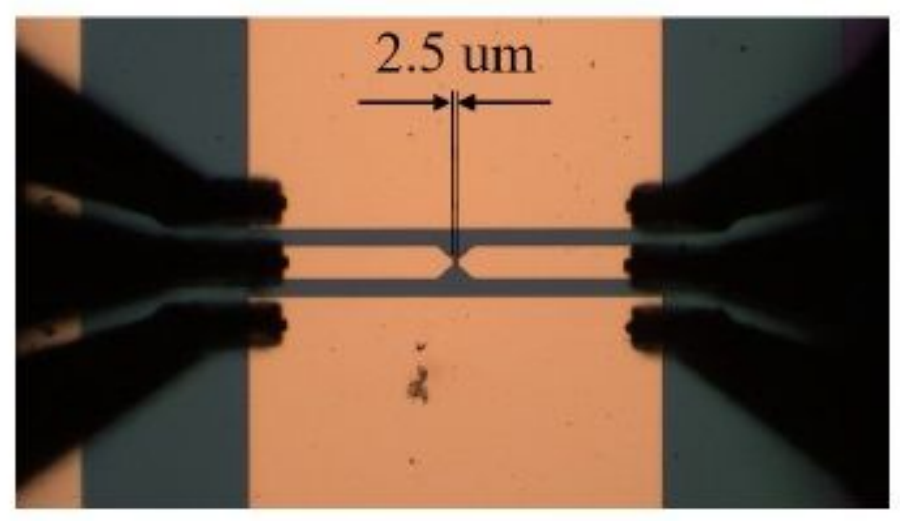

(a)

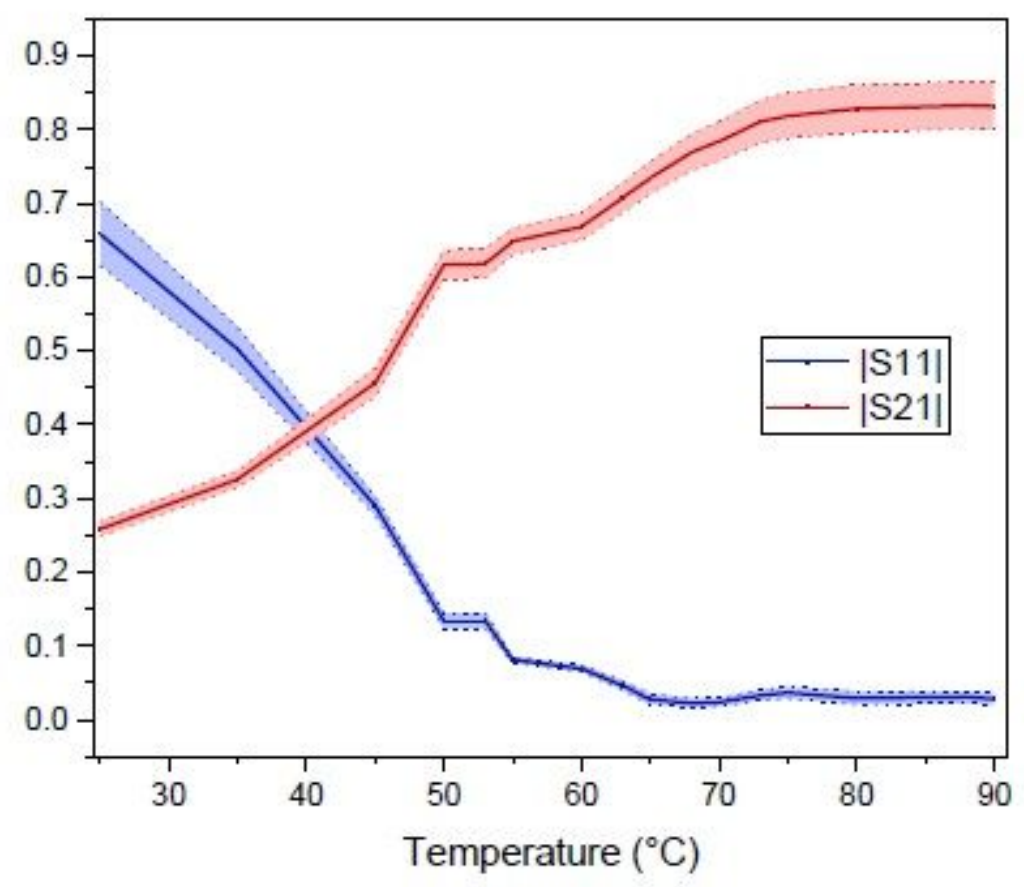

(b)

\section{Figure 3}

interrupted CPW by VO2. (a) under the microscope, (b) S parameters magnitude between the two ports across the CPW vs. temperature 


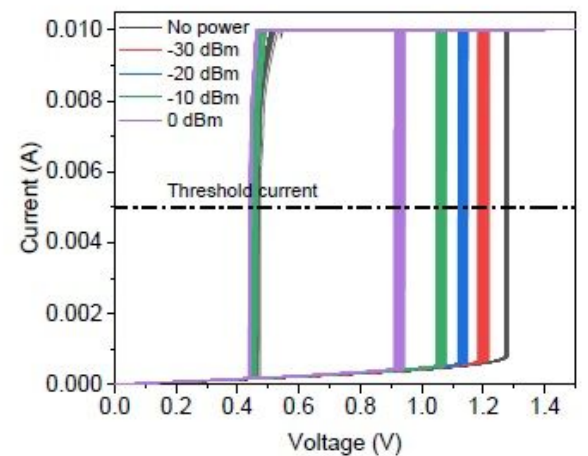

(a)

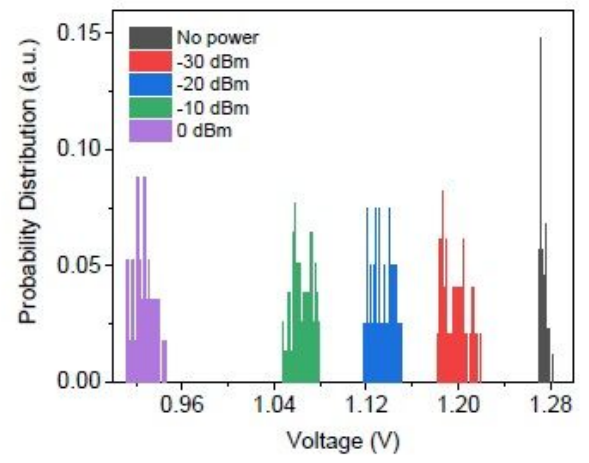

(d)

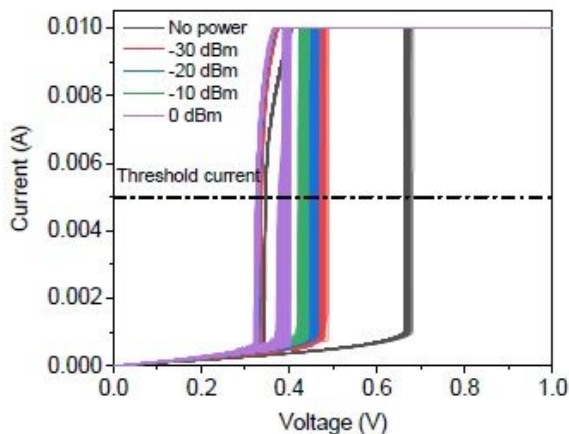

(b)

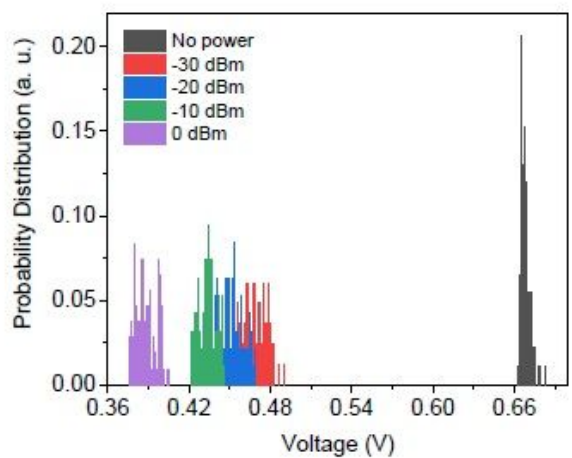

(e)

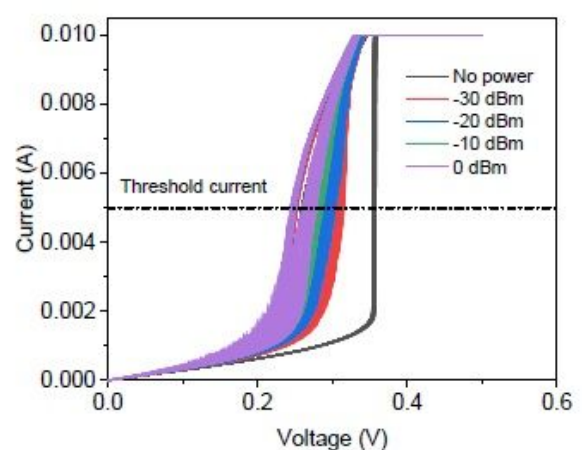

(c)

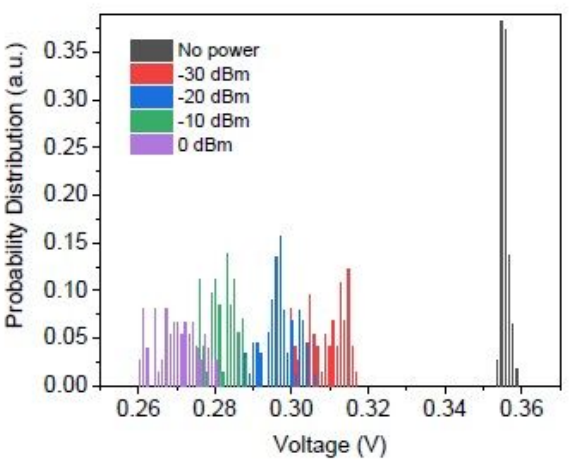

(f)

\section{Figure 4}

(a) - (c) static I-V characteristics of VO2 with respect to different levels of external power, at (a) room temperature, (b) $40^{\circ} \mathrm{C}$, and (c) $50^{\circ} \mathrm{C}$. (d) - (f) probability distribution of IMT threshold voltage, at (d) room temperature, (e) $40^{\circ} \mathrm{C}$, and (f) $50^{\circ} \mathrm{C}$. Elevated temperatures make major shifts in the threshold voltage, while the effect of the external power is maintained in all cases. The temperature role is mainly on the resolution between the curves at different power levels. 


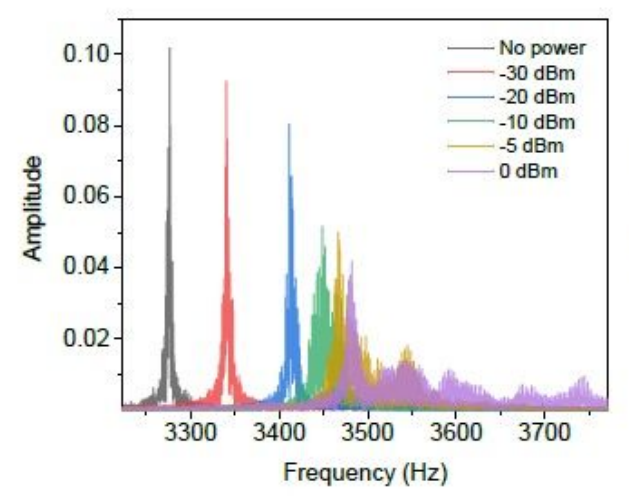

(a)

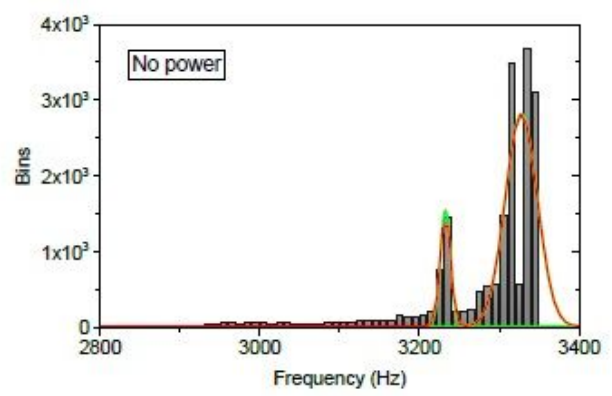

(d)

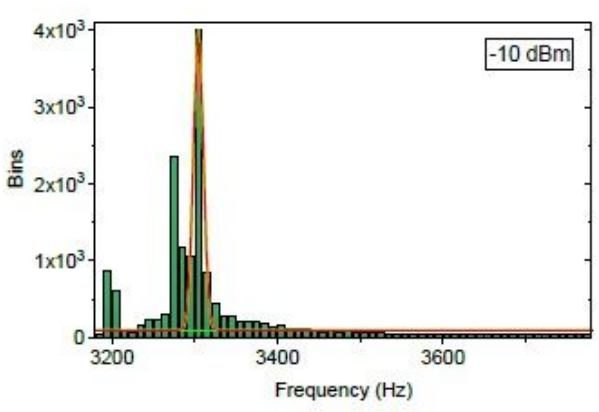

(g)

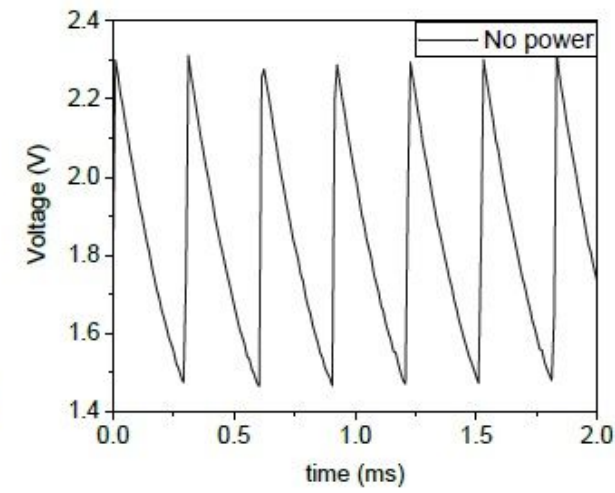

(b)

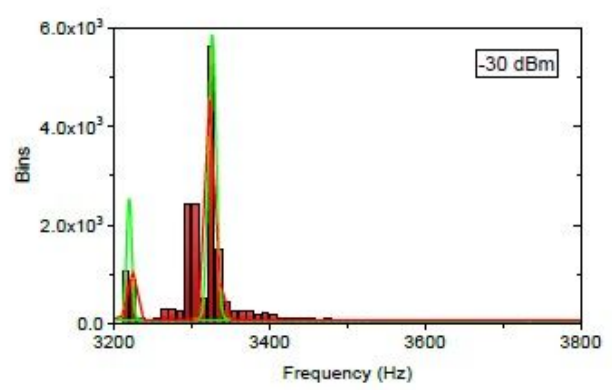

(e)

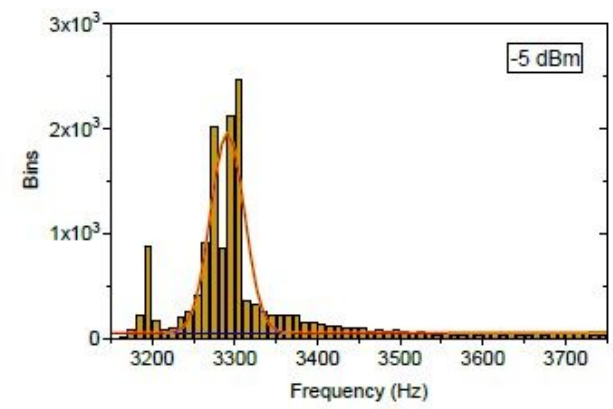

(h)

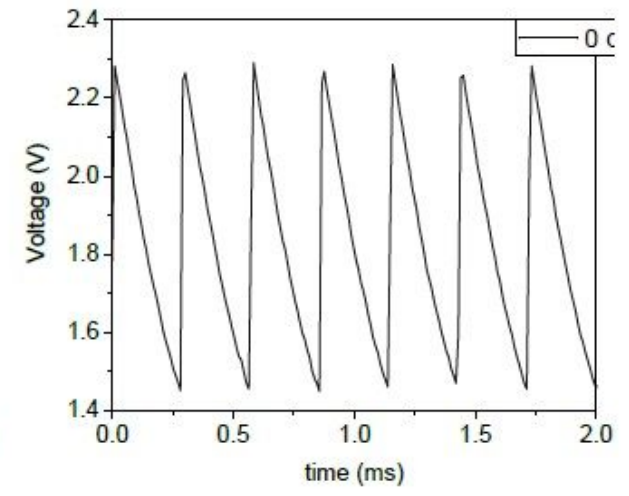

(c)

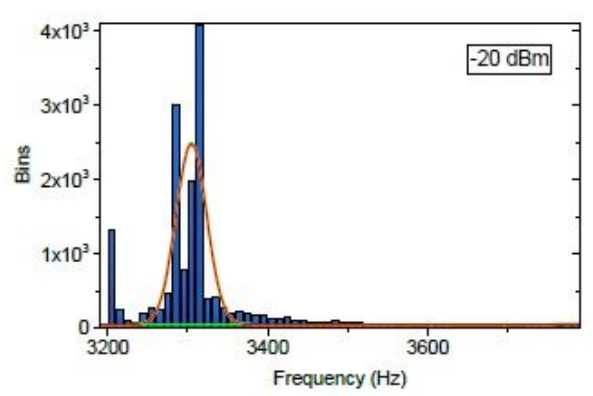

(f)

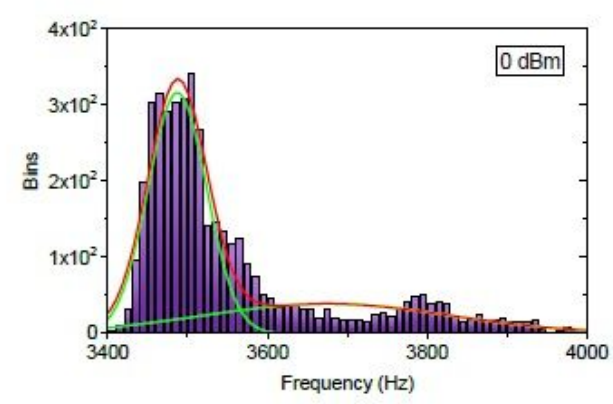

(i)

Figure 5

Frequency of oscillations based on applied power (a) FFT of output waveforms with respect to the applied power, (b) and (c) time domain waveforms for "no power" and "0 dBm" exposure, (d)-(i) distributions for the frequency of oscillations based on the emitted power from the first port. 


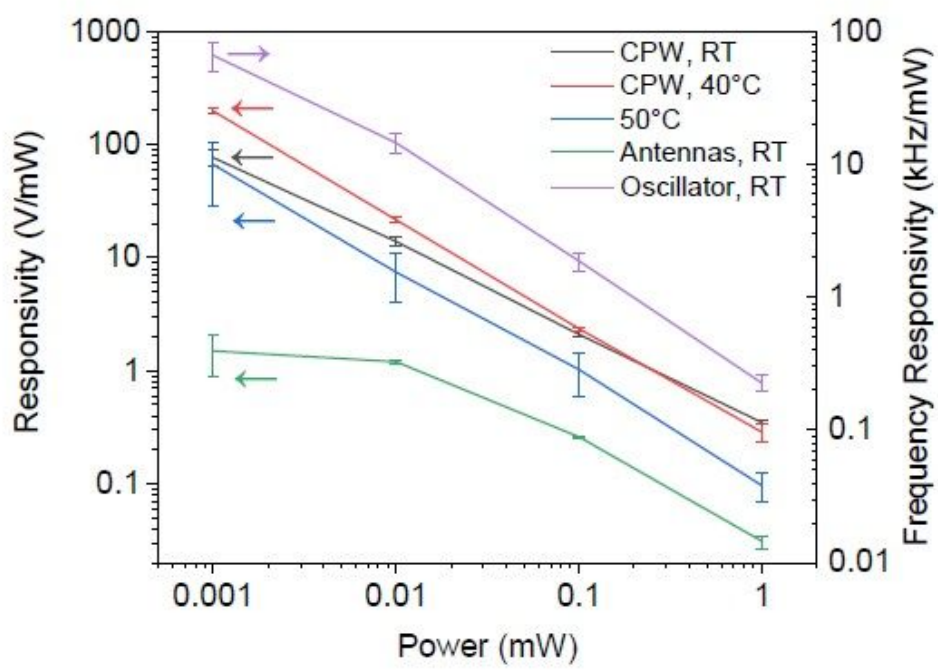

(a)

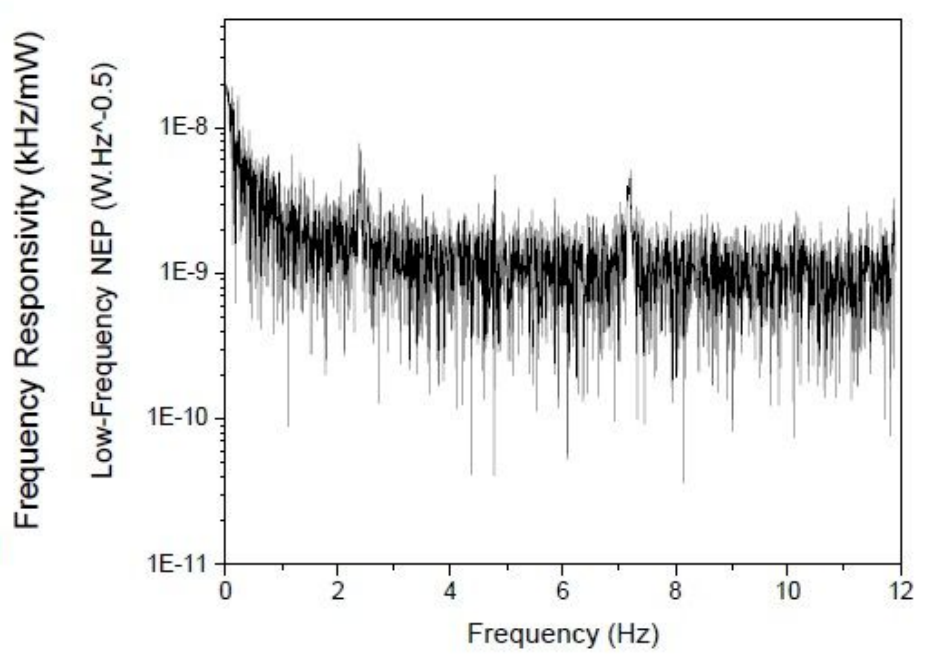

(b)

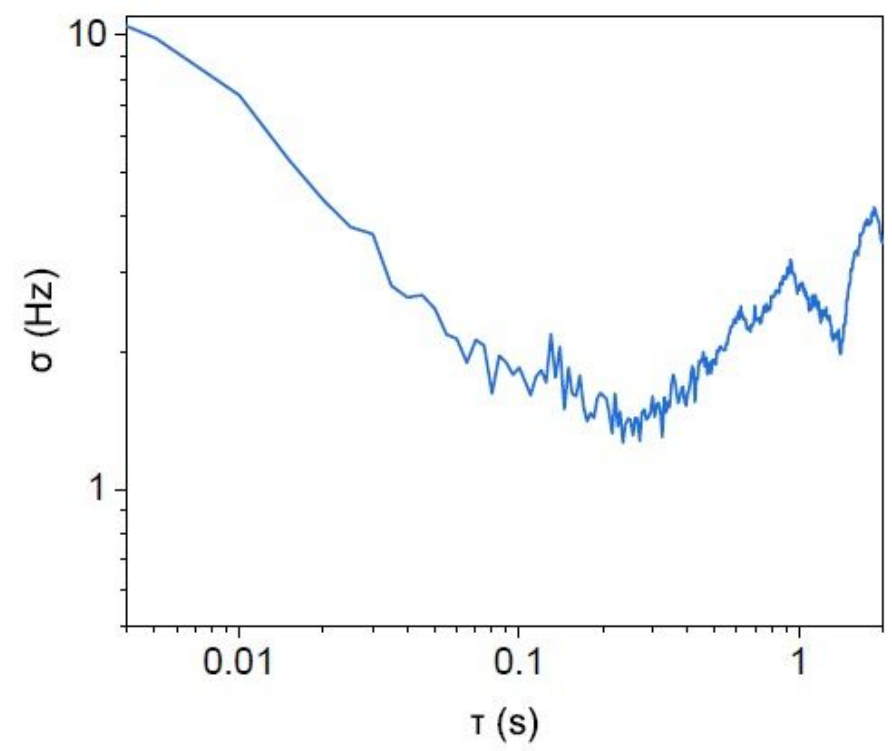

(c)

\section{Figure 6}

figure of merit for the proposed VO2-based sensor (a) DC and frequency responsivity due to the shift in the IMT threshold voltage for the mentioned configurations based on the emitted power from the first port, (b) Noise equivalent power of the CPW devices at very low frequencies, (c) Allan deviation calculation on the frequency vs time measurements of the oscillator over a long duration of time, no external power applied

\section{Supplementary Files}

This is a list of supplementary files associated with this preprint. Click to download.

- Supplementary.pdf 